\title{
First Observation of Laser-Driven Particle Acceleration in a Semi-Infinite Vacuum Space
}



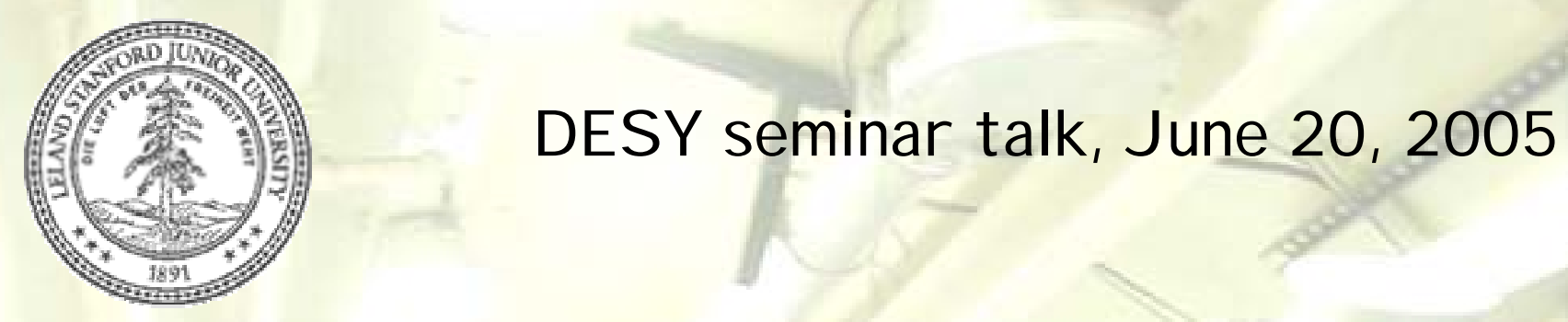

Stanford Linear Accelerator Center

\title{
First Observation of Laser-Driven Particle Acceleration in a Semi-Infinite Vacuum Space
}

\author{
T. Plettner, R.L. Byer
}

E.L. Ginzton Laboratories, Stanford University

E. Colby, B. Cowan, C.M. Sears, R.H. Siemann, J.E. Spencer $A R D B, S L A C$ 


\section{Contents of the presentation}

\section{Overview}

- Motivation for laser-driven particle acceleration

- The physics concept

- Overview of the LEAP experiment

\section{The LEAP experiment}

- Important components of the LEAP experiment

- The simplified acceleration geometry

- The disposable boundary approach

- The insertion of an IFEL as a timing monitor

3. Results

- Confirmation of the Lawson-Woodward Theorem

- $\quad$ Proof of the linear dependence on the electric field

- Expected polarization dependence

- Expected slow dependence on laser crossing angle

4. Future experiments

- Test on different boundaries

- Measurements below the laser damage threshold

- E163 at SLAC

5. Summary 


\section{Motivation}

\section{historical trend of high energy physics experiments}
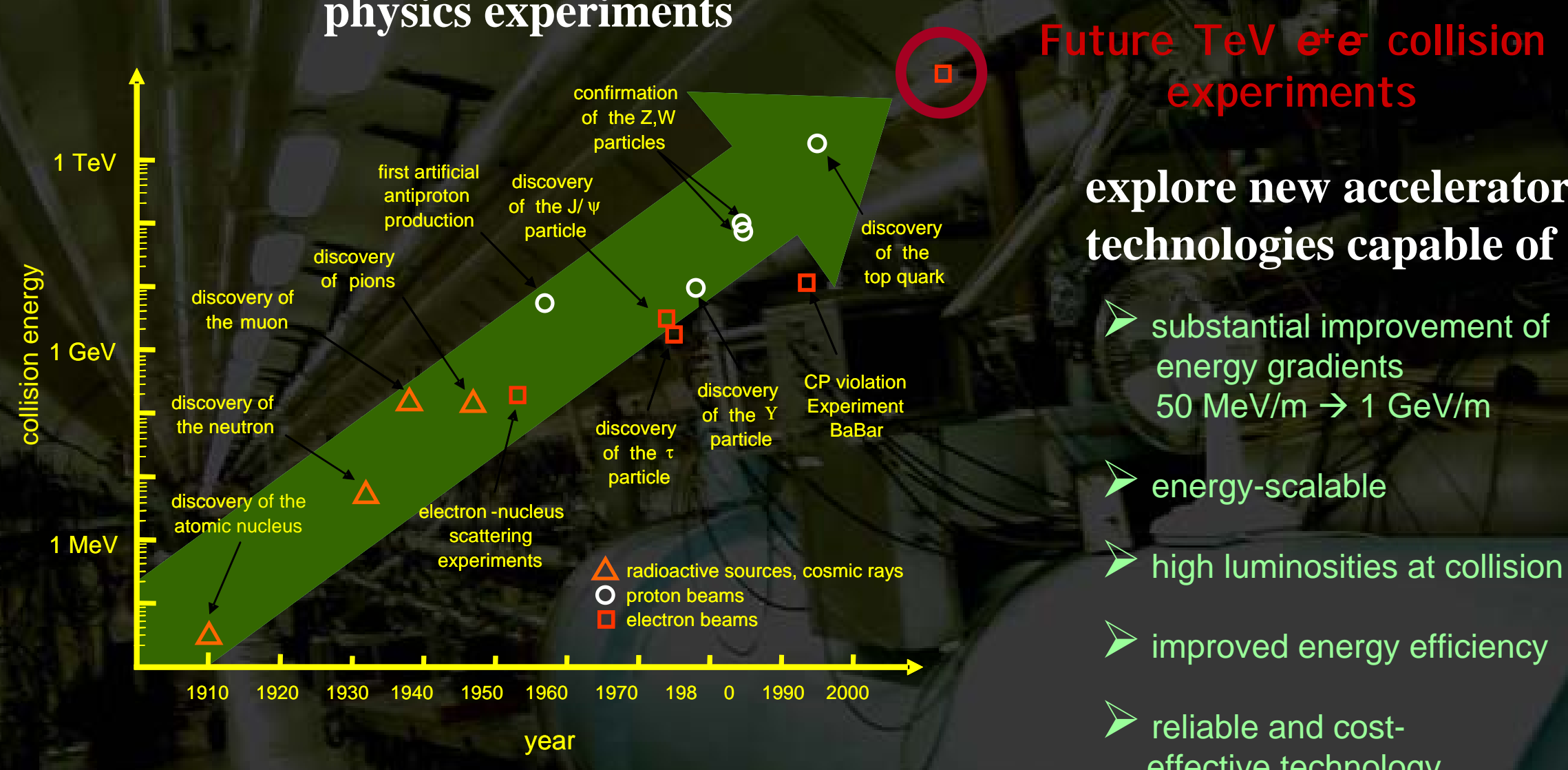

\section{explore new accelerator}

technologies capable of

$>$ substantial improvement of energy gradients $50 \mathrm{MeV} / \mathrm{m} \rightarrow 1 \mathrm{GeV} / \mathrm{m}$

$\gg$ energy-scalable

$>$ high luminosities at collision

improved energy efficiency

reliable and costeffective technology 


\section{Laser driven particle acceleration in vacuum}

\section{Characteristics}

$>$ Crossing laser beams in vacuum

- Longitudinal electric field of the laser pattern responsible for particle acceleration

$>$ Dielectric accelerator structure

- $\sim 1 \mathrm{~g} / \mathrm{cm}^{2}$ damage threshold with ultra-short ne ar IRlasers

- with $100 \mathrm{fsec}$ pulses peakfields of $10 \mathrm{GV} / \mathrm{m}$ possible

- a $1 \mathrm{GeV} / \mathrm{m}$ gradient structure is possible 1

$>$ The acceleration is linear

- we are interested in a line ar acceleration process $G \sim E_{\|}$

The electrons stay in vacuum

- no beam deterioration from scattering with matter

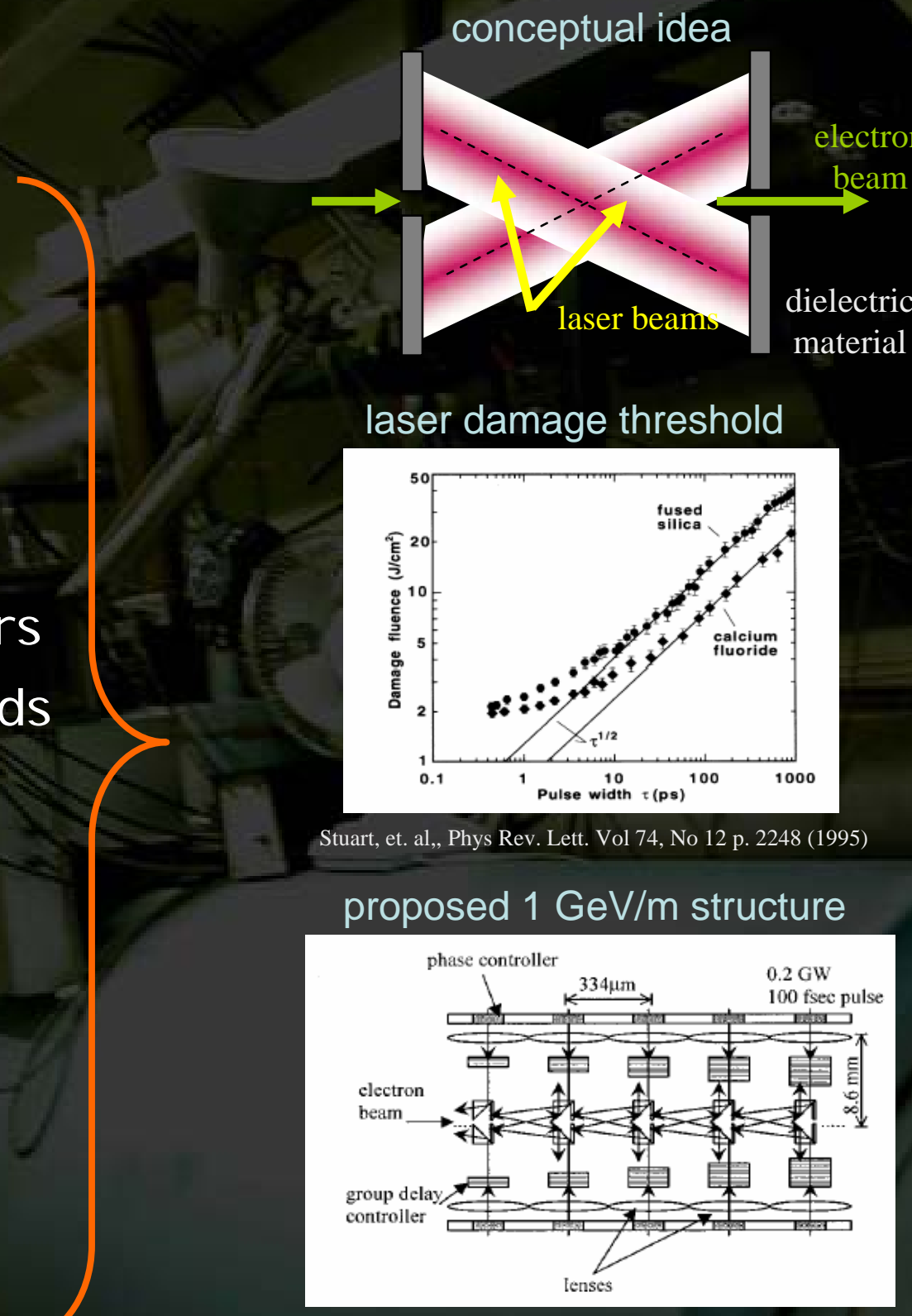

Y.C. Huang, et al, Appl. Phys. Lett. 68 (6) (1996) 753. 


\section{The basic physics concept}

Example: Longitudinal electric field from crossed laser beams *

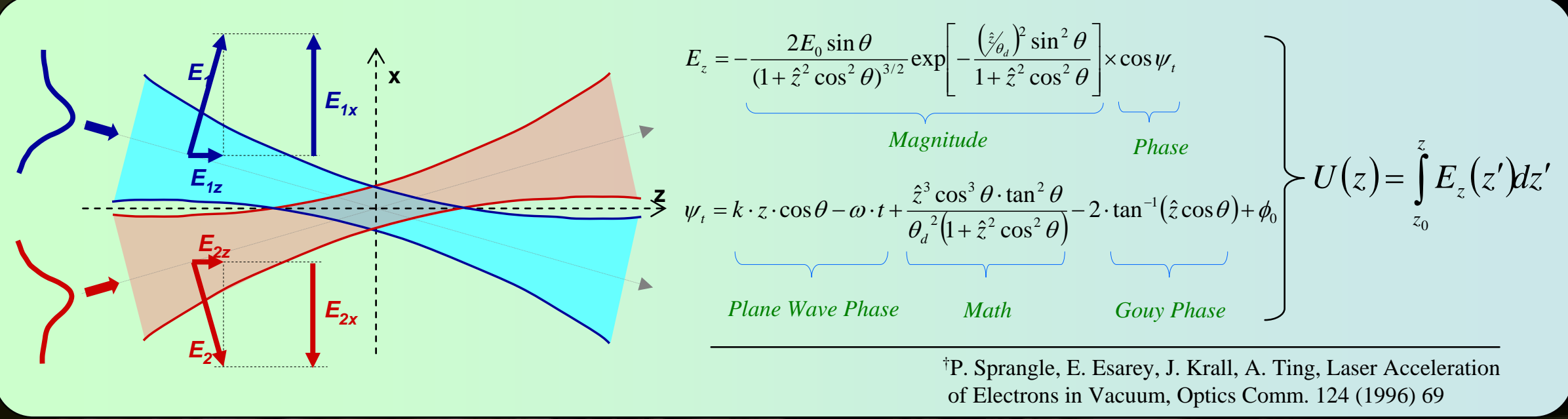

Plot of the longitudinal E-field

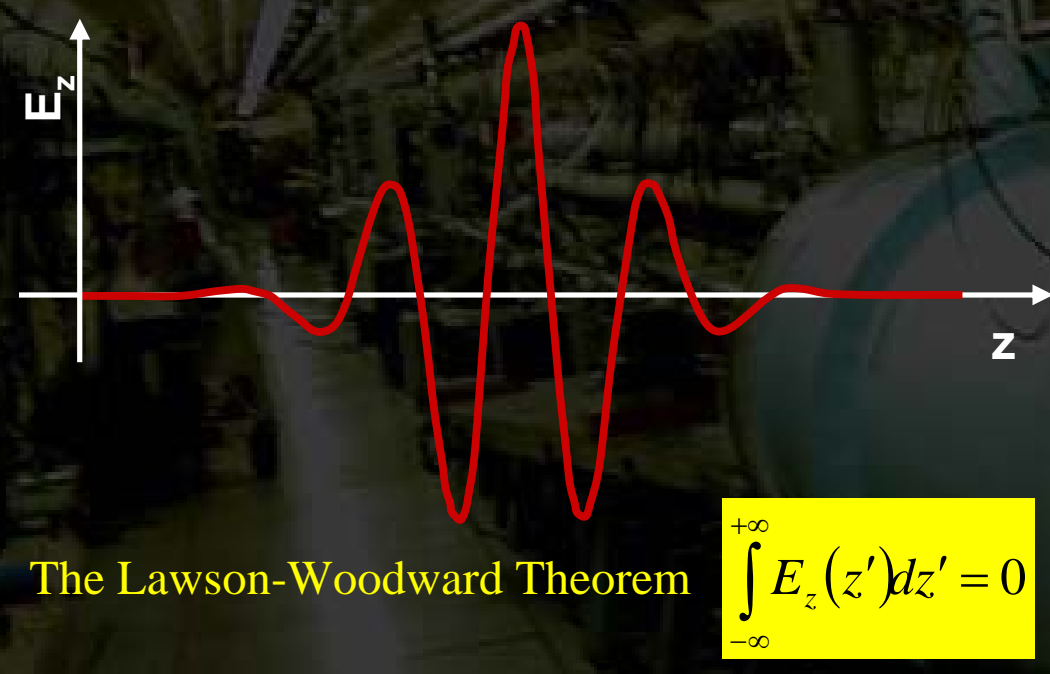

Limit the laser-electron interaction

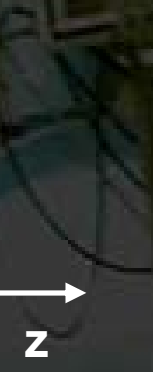

laser beams

dielectric boundary

$$
\int_{z_{1}}^{z_{2}} E_{z}\left(z^{\prime}\right) d z^{\prime} \neq 0
$$

*For an alternate picture of particle acceleration see Z. Huang, G. Stupakov, M. Solotorev, "Calculation and optimization of laser acceleration in vacuum", Phys. Rev. ST AB 7011302 (2004) 


\section{The SCA-FEL Facility}
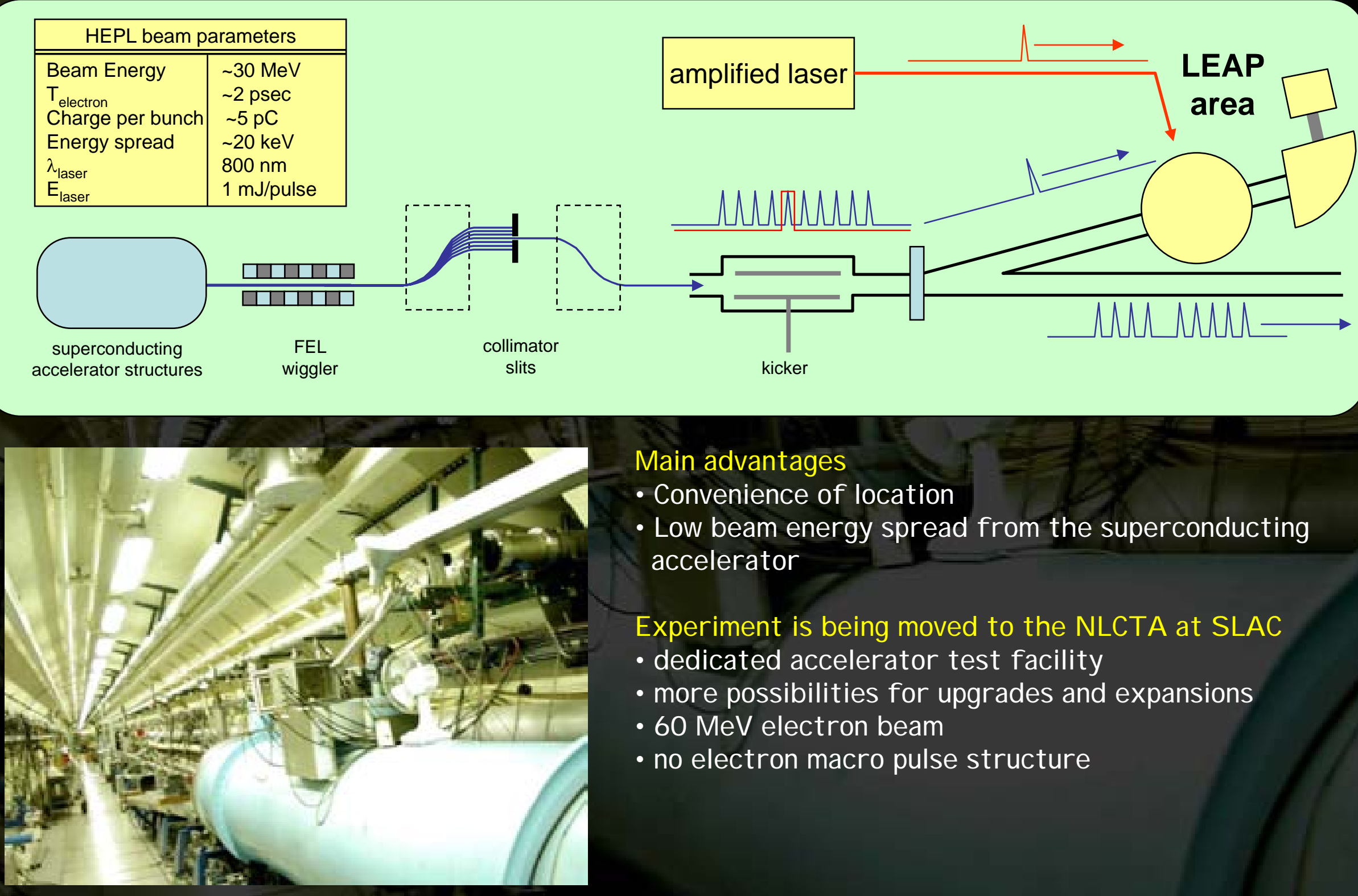

Main advantages

- Convenience of location

- Low beam energy spread from the superconducting accelerator

Experiment is being moved to the $\mathcal{N} L C \mathcal{A}$ at $S \mathcal{L A C}$

- de dicated accelerator test facility

- more possibilities for upgrades and expansions

- 60 MeVelectron be am

- no electron macro pulse structure 


\section{Contents of the presentation}

\section{Overview}

- Motivation for laser-driven particle acceleration

- The physics concept

- Overview of the LEAP experiment

\section{The LEAP experiment}

- Important components of the LEAP experiment

- The simplified acceleration geometry

- The disposable boundary approach

- The insertion of an IFEL as a timing monitor

\section{Results}

- Confirmation of the Lawson-Woodward Theorem

- Proof of the linear dependence on the electric field

- Expected polarization dependence

- Expected slow dependence on laser crossing angle

\section{Future experiments}

- Test on different boundaries

- Measurements below the laser damage threshold

- E163 at SLAC

\section{Summary}




\section{Problems with the old LEAP cell}

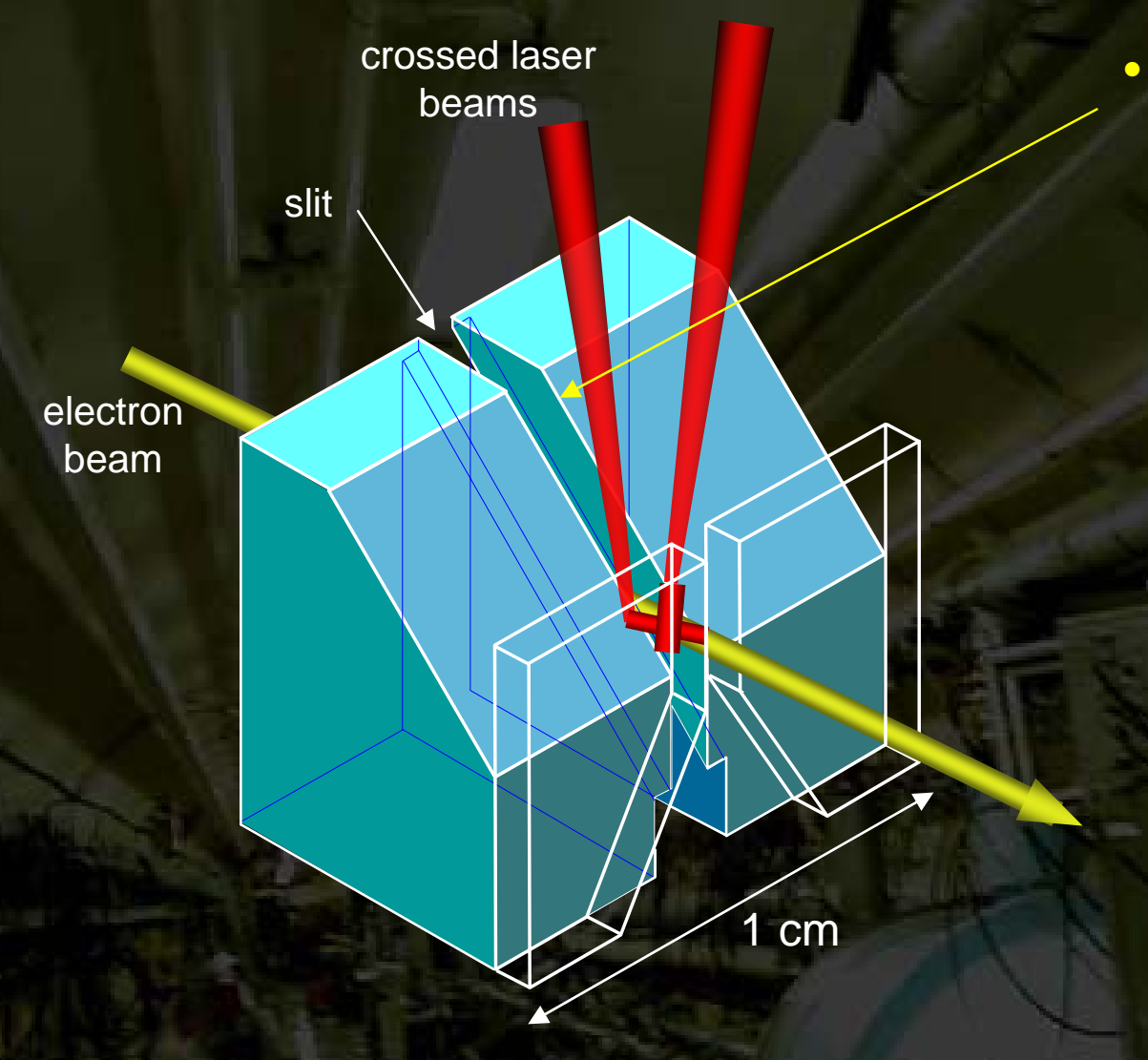

- HR coated fused silica with sharp edges in vacuum $\rightarrow$ reduction of laser damage threshold to $\sim 1 / 4 \mathrm{~J} / \mathrm{cm}^{2}$

$\rightarrow$ max. energy gain of $\sim 20 \mathrm{keV}$

- moving part of accelerator cell for variable slit $\rightarrow$ internal alignment uncertainty once in operation $\rightarrow$ very difficult e-beam alignment (heavy loss of beam through a 10 micron slit

$\rightarrow$ poor transmission through the $10 \mu \mathrm{m}$ slit

- crossed laser beams

$\rightarrow$ difficult alignment inside the cell space

$\rightarrow$ optical phase uncertainty

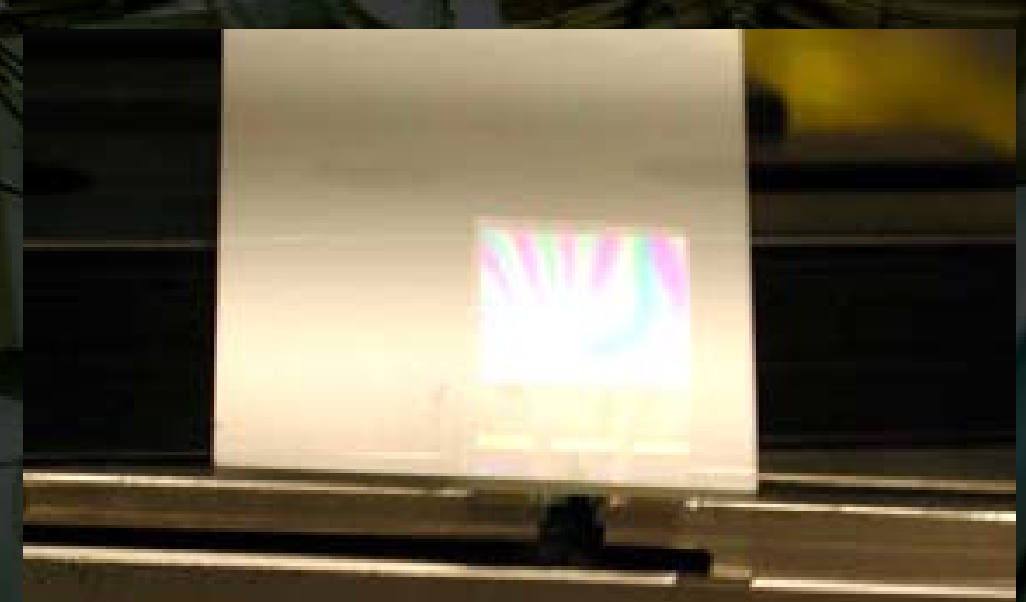

Interferometric alignment of prism surfaces 


\section{The simplified acceleration geometry and the disposable boundary}

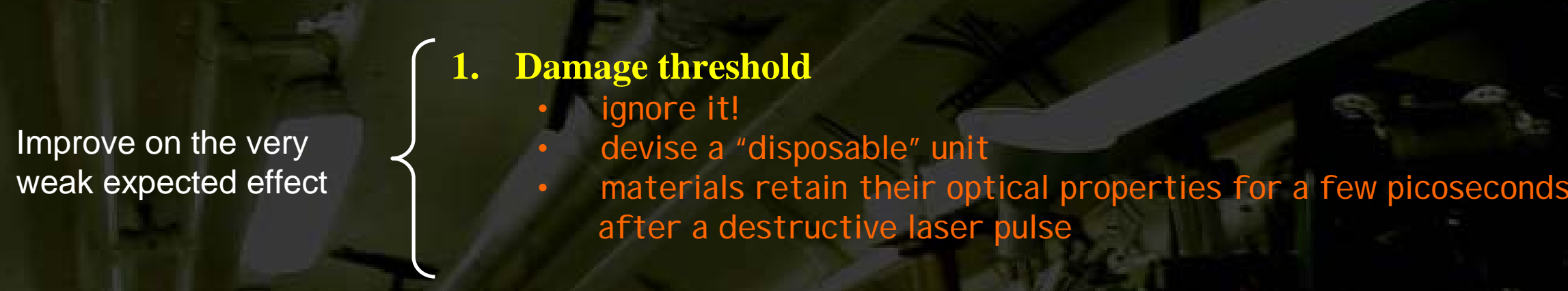

\section{Improve on}

- Operation tolerances

- Poor reliability

- Ease of operation

\section{Cell geometry}

- simplify to one semi-infinite boundary

- make boundarythin enough to rune-beam through it

- make boundary movable to present a newsurface for each laser shot

3. Crossed laser beams

- twolaser beams too difficult? $\rightarrow$ eliminate one of them

- no more opticalphase uncertainty problems

negligible transversedeflection forces

Conceptual drawing of the improved setup
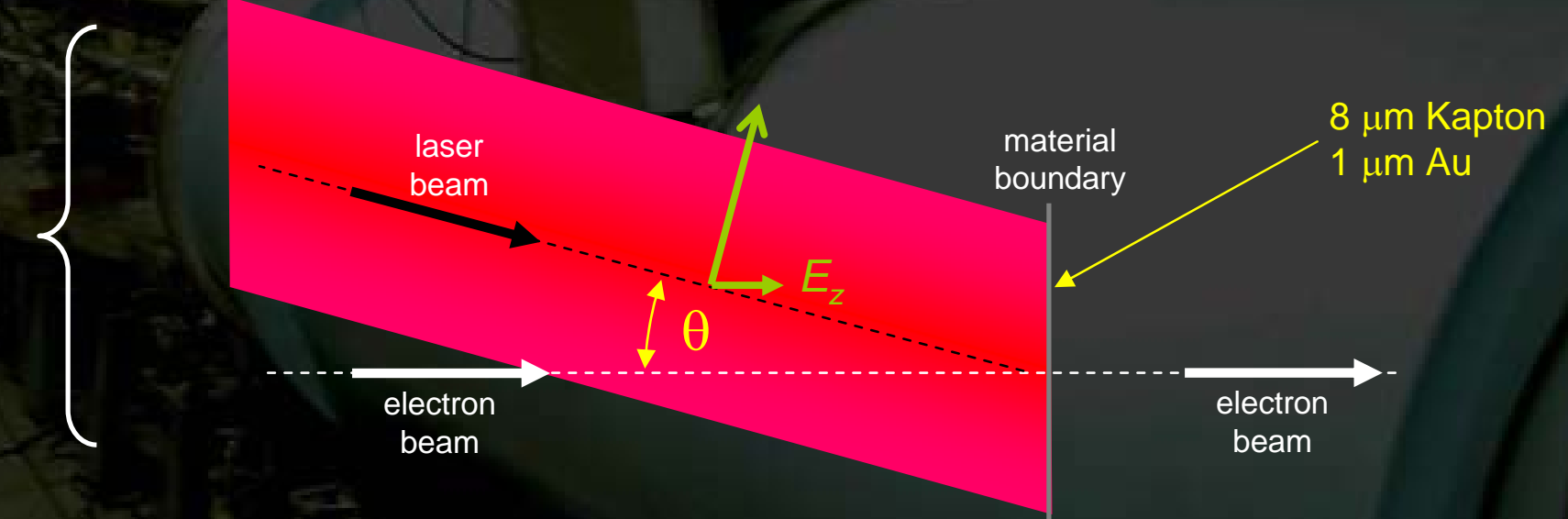


\section{The reflective boundary tape drive}

stepper motor

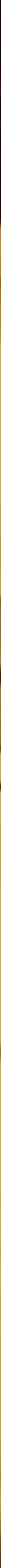




\section{Dual IFEL-ITR experiment operation}

Use the I FEL as a psec-resolution timing diagnostic

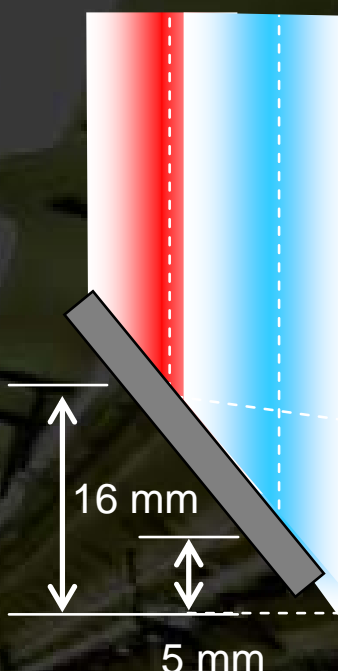

Regen average power: $\sim 1 / 2 \mathrm{~W}$

FWHM laser spot size: $50 \mu \mathrm{m}$

FWHM laser pulse: $2.0 \mathrm{psec}$

Xing angle for ITR: $16 \mathrm{mrad}$

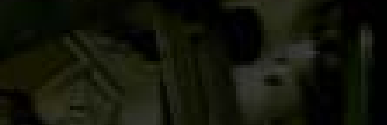

(1)
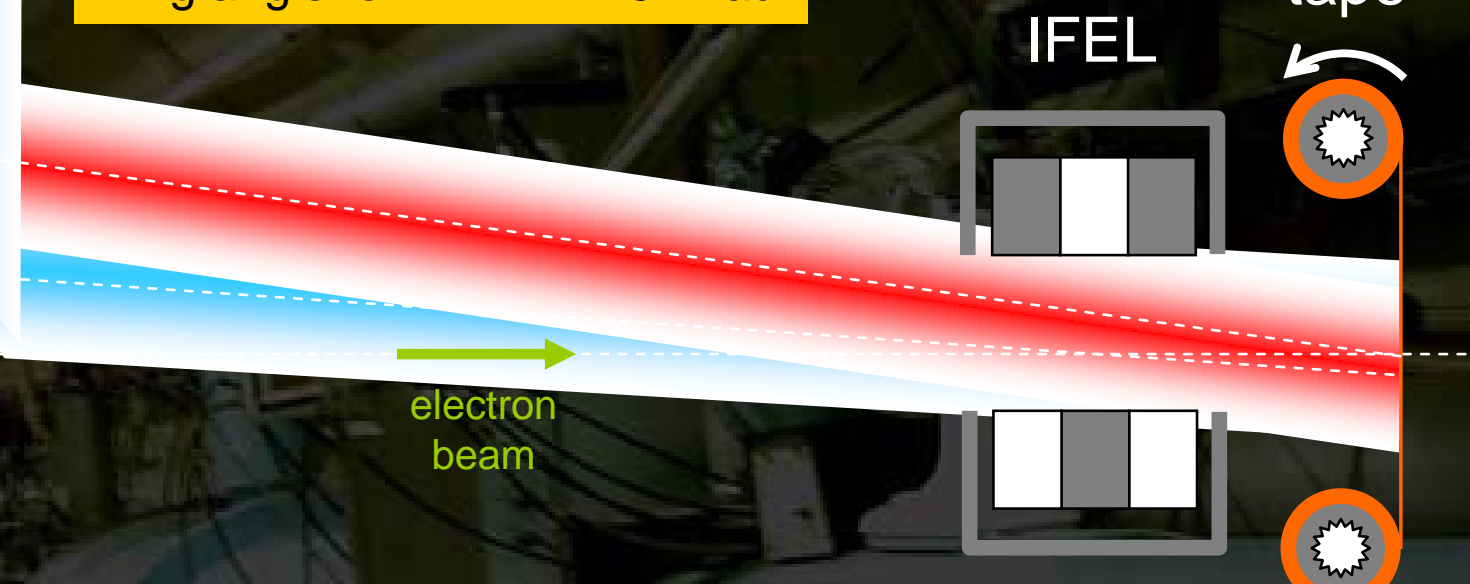

Important features

- IFEL 10 cm upstream of tape boundary

- laser and electron beams cannot be simultaneously aligned for both the IFEL and the tape

- IFEL and ITR timing conditions are almost identical

- The IFEL produces a larger and easier to detect signal

See publication by C.M. Sears et al, "High Harmonic IFEL at 800 nm“ (TPAE029) 


\section{Top view of breadboard}

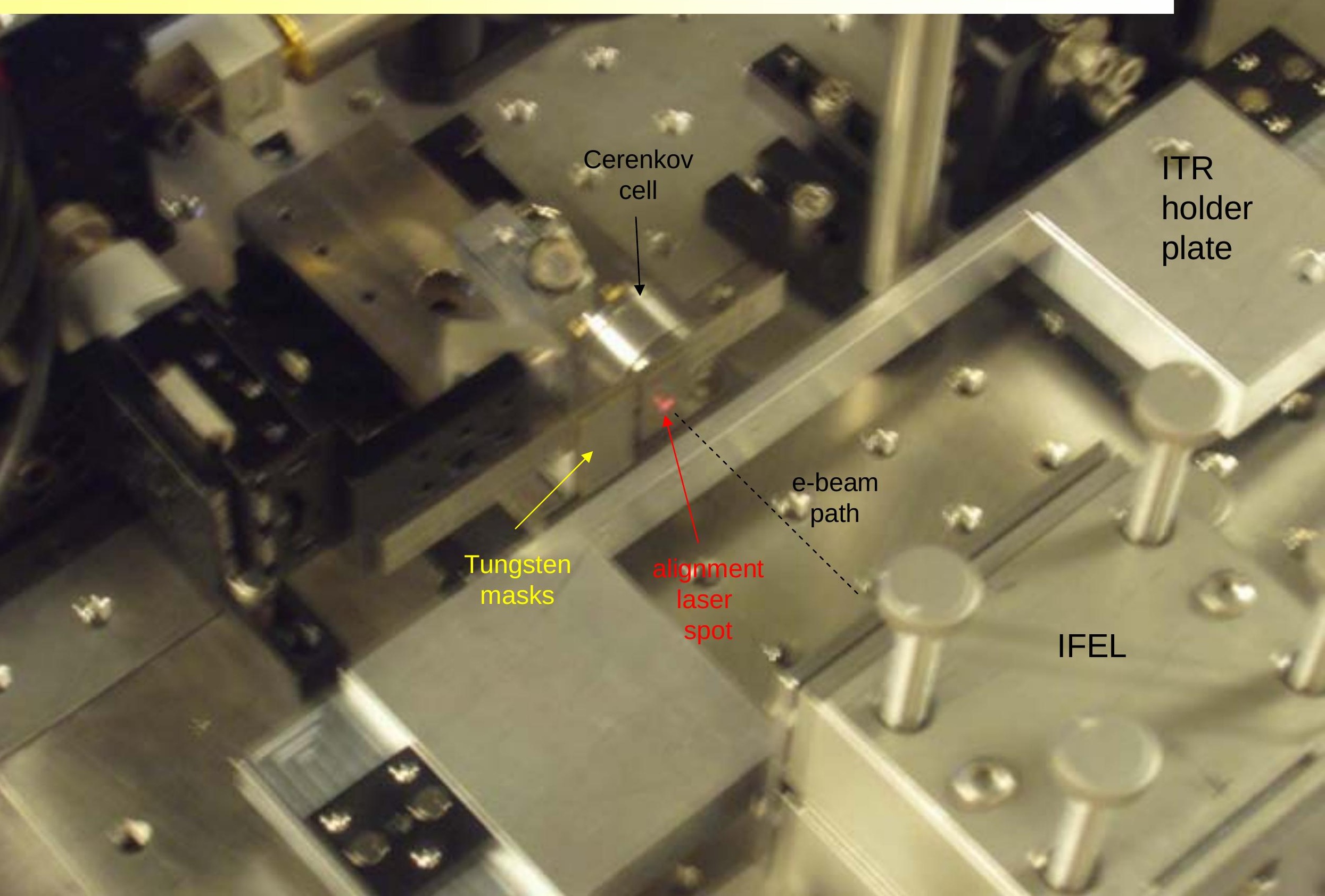


Back-of-ITR downstream

Puckùp mirror $\quad \therefore \quad$ Cerenkov

$(\mathrm{CH}, 24)$

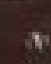

,

Cerènkôv ',

: cèll

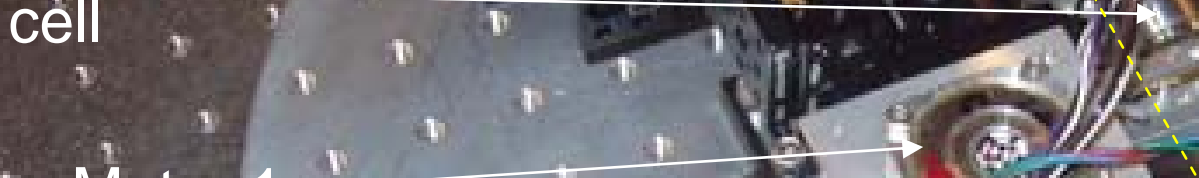

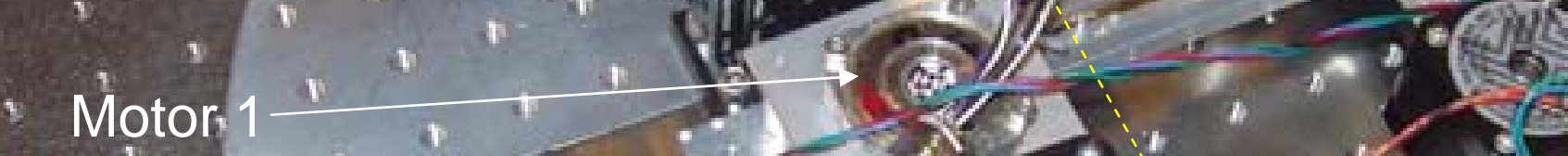

( $(\mathrm{CH}$ 21

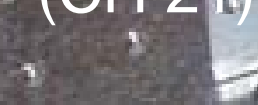

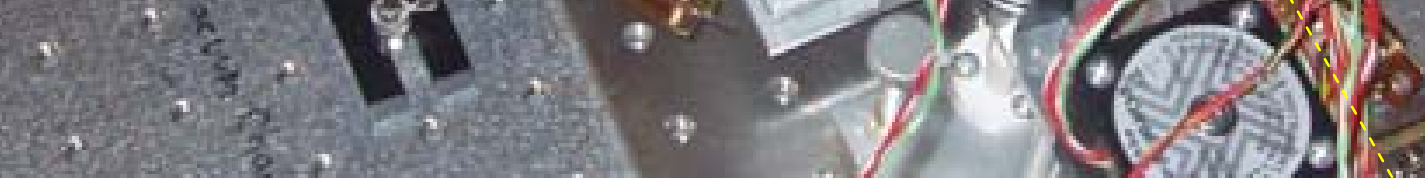

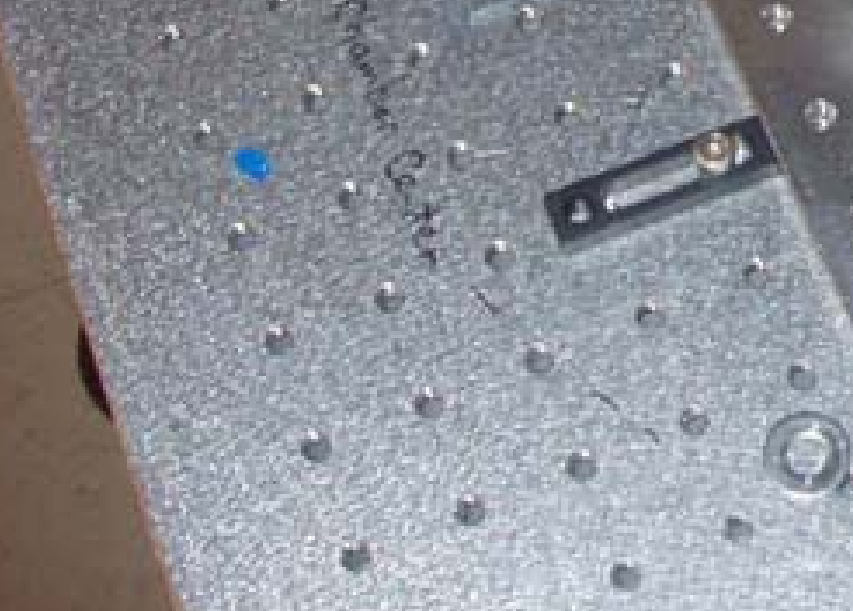




\section{Contents of the presentation}

\section{Overview}

- Motivation for laser-driven particle acceleration

- The physics concept

- Overview of the LEAP experiment

\section{The LEAP experiment}

- Important components of the LEAP experiment

- The simplified acceleration geometry

- The disposable boundary approach

- The insertion of an IFEL as a timing monitor

3. Results

- Confirmation of the Lawson-Woodward Theorem

- Proof of the linear dependence on the electric field

- Expected polarization dependence

- Expected slow dependence on laser crossing angle

4. Future experiments

- Test on different boundaries

- Measurements below the laser damage threshold

- E163 at SLAC

5. Summary 


\section{Expected observations at the energy spectrometer}

Electrons are not optically bunched

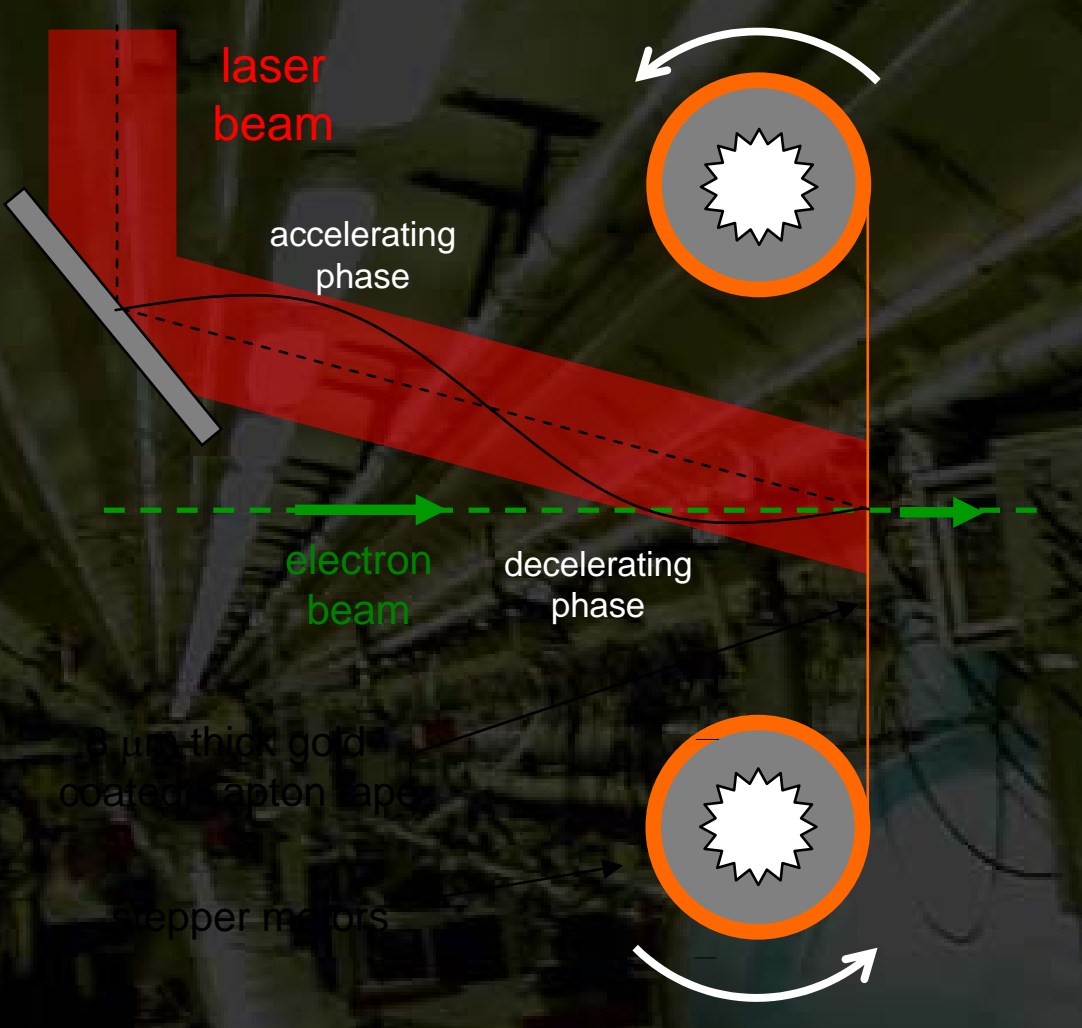

Electrons are distributed evenly over all the laser optical phases

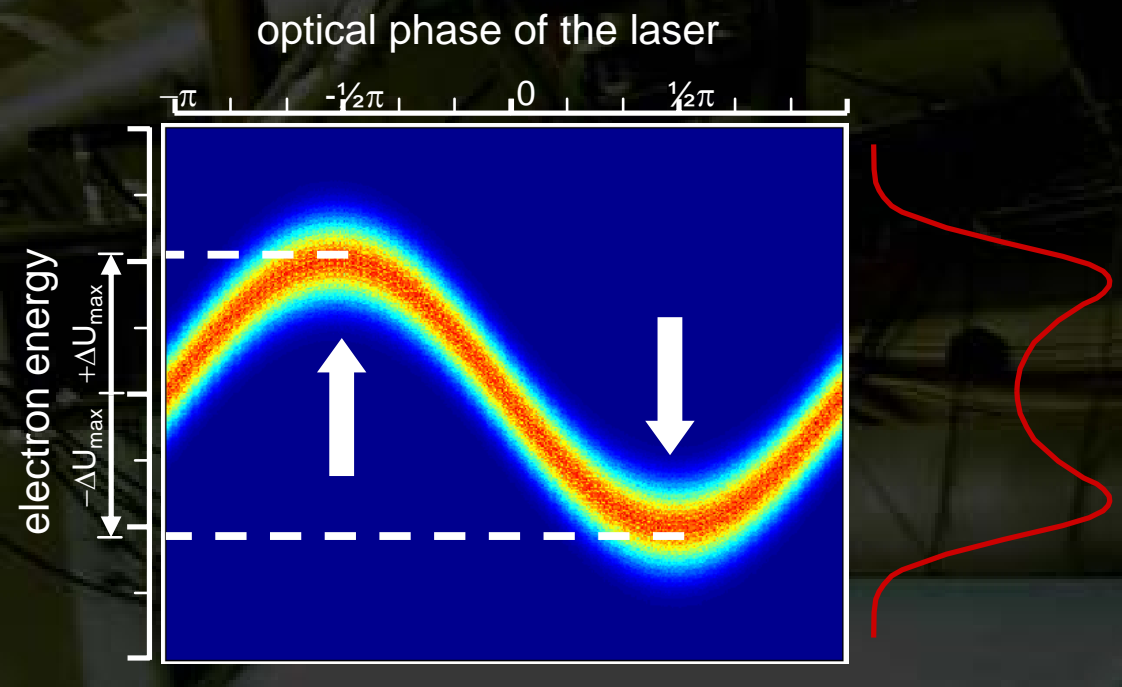

We expect to observe a broadening of the initial energy spread of the electron beam that is related to the modulation strength of the laser 


\section{Data acquisition}

Data was taken as laser time scans

- timing of laser shots scanned over a 20-30 psec timing window

- Laser was randomly toggled on and off

- we lookfor changes of the energy width be twe en laser-on and laser-off data

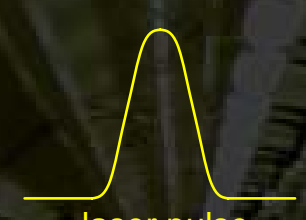

laser pulse

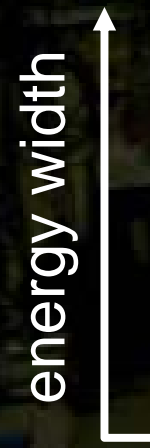

laser time
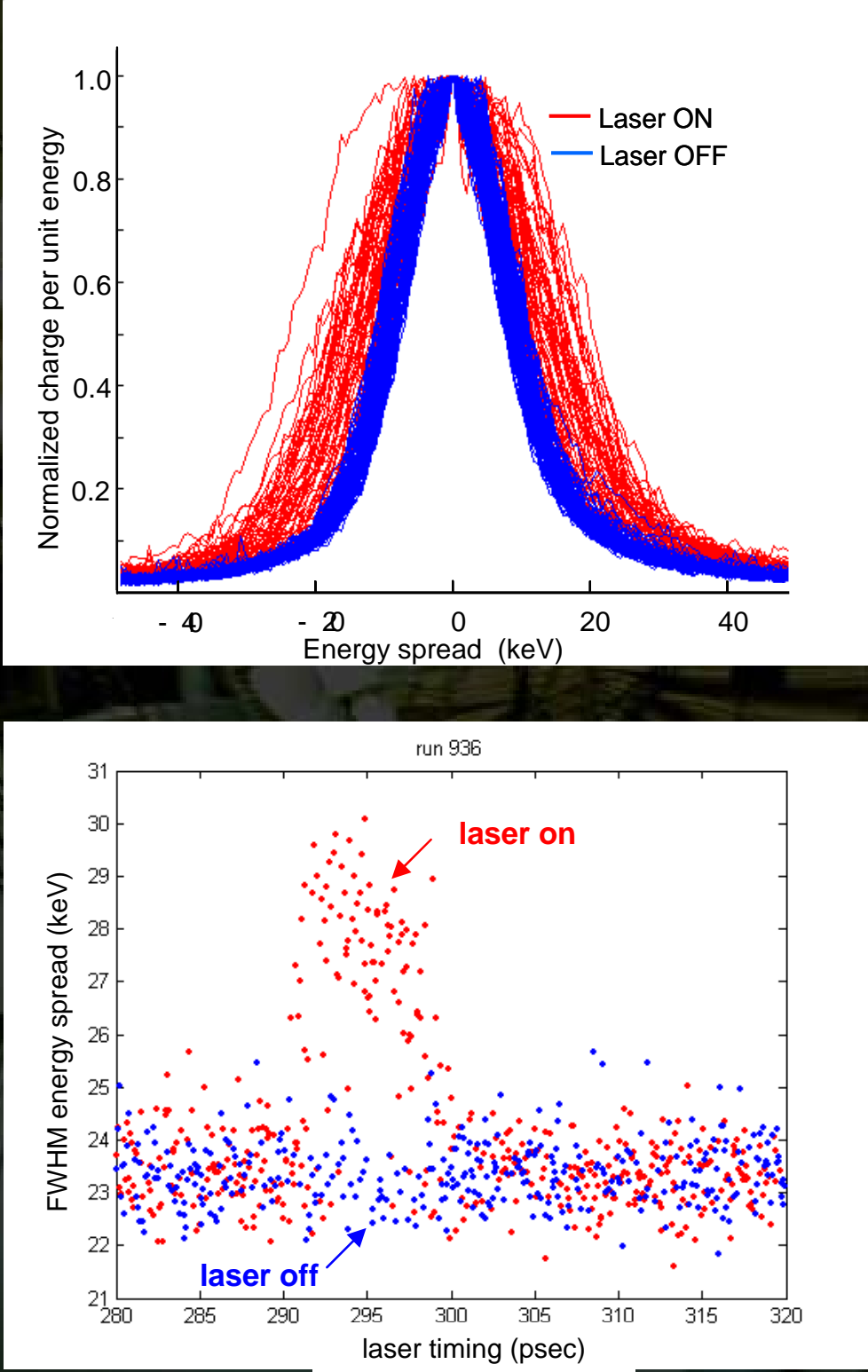


\section{Expected dependence of the laser-driven energy modulation}

effects we studied during the accelerator run
1. The Lawson-Woodward Theorem

2. Laser peak electric field

3. Laser polarization

4. Laser crossing angle

Expected dependences

Numerical integration of the longitudinal electric field along the e-beam trajectory

$$
U=\int_{-\infty}^{0} e \cdot E_{z}\left(z^{\prime}\right) d z^{\prime}
$$
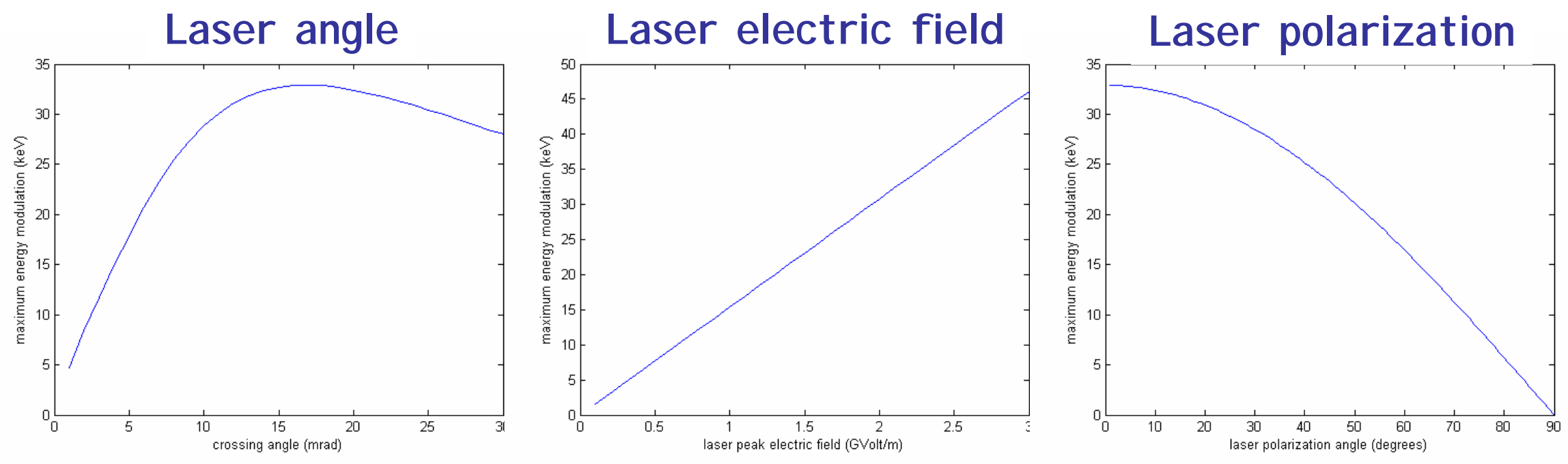

$|U| \propto\left|\sin \alpha \cdot Z_{\text {slippage }}\right|$

$$
|U| \propto|E|_{\text {laser }}
$$

$$
|U| \propto|\cos \phi|
$$




\section{Confirmation of the Lawson-Woodward Theorem}
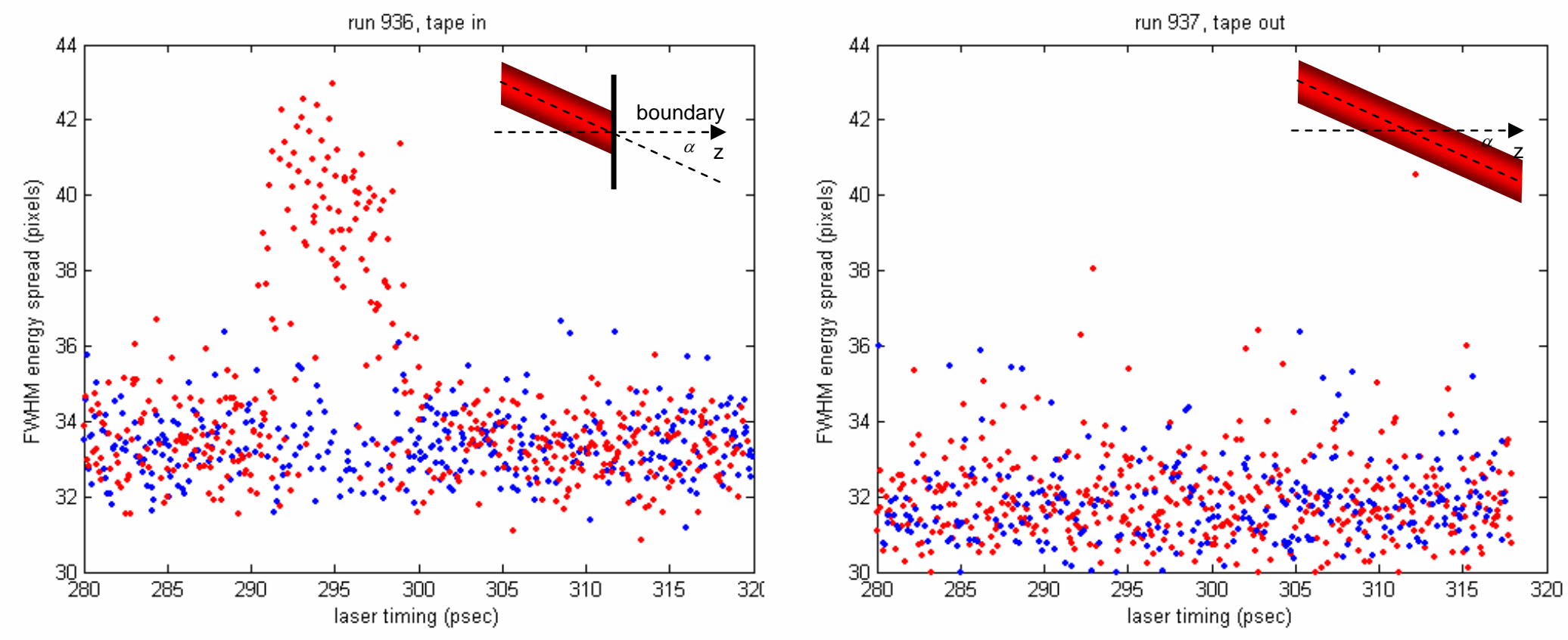

This confirms:

1. The Lawson-Woodward The orem

2. No interaction from the I FEL 


\section{Laser electric field strength dependence}

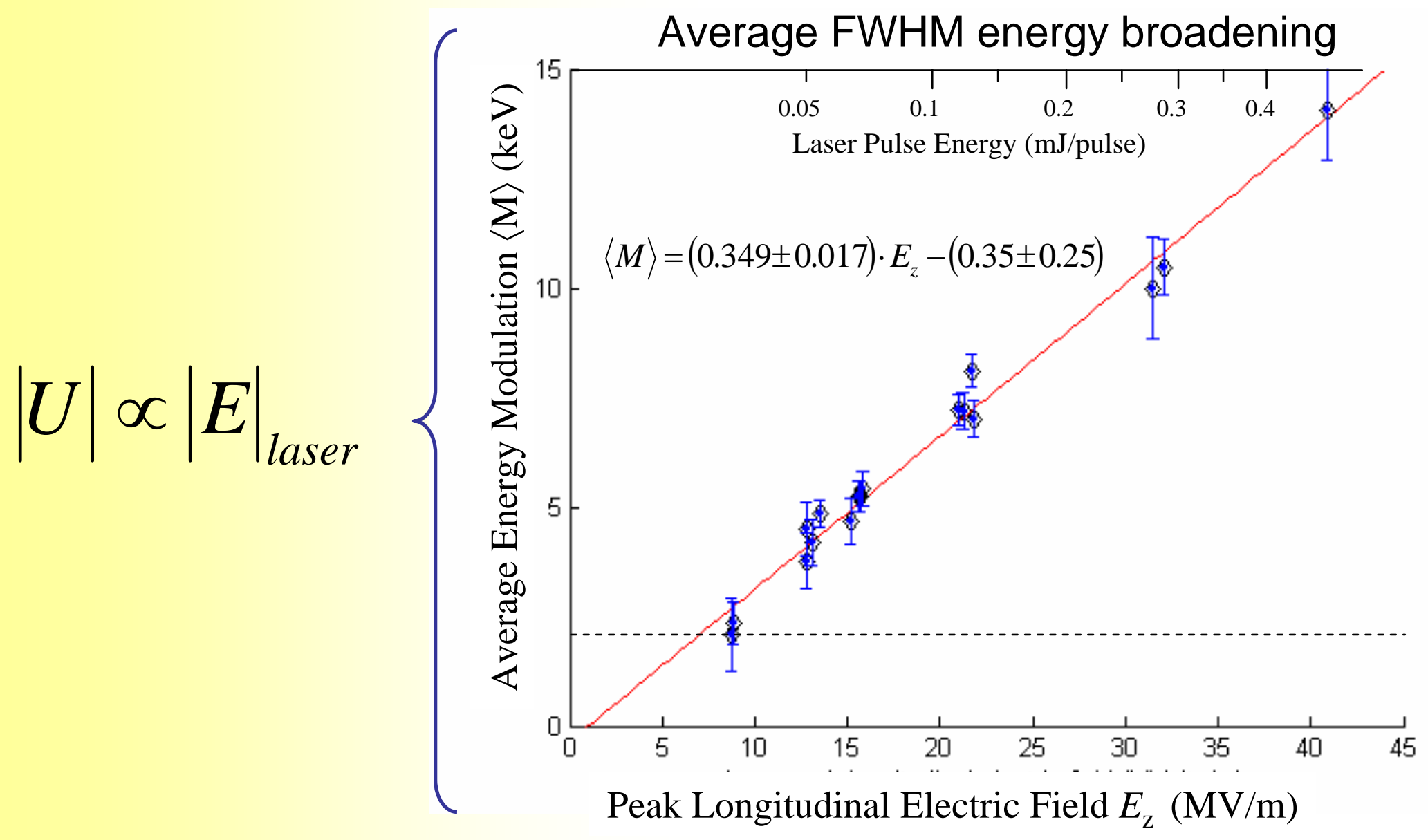




\section{Laser polarization angle dependence}

$|U| \propto|\cos \phi|$

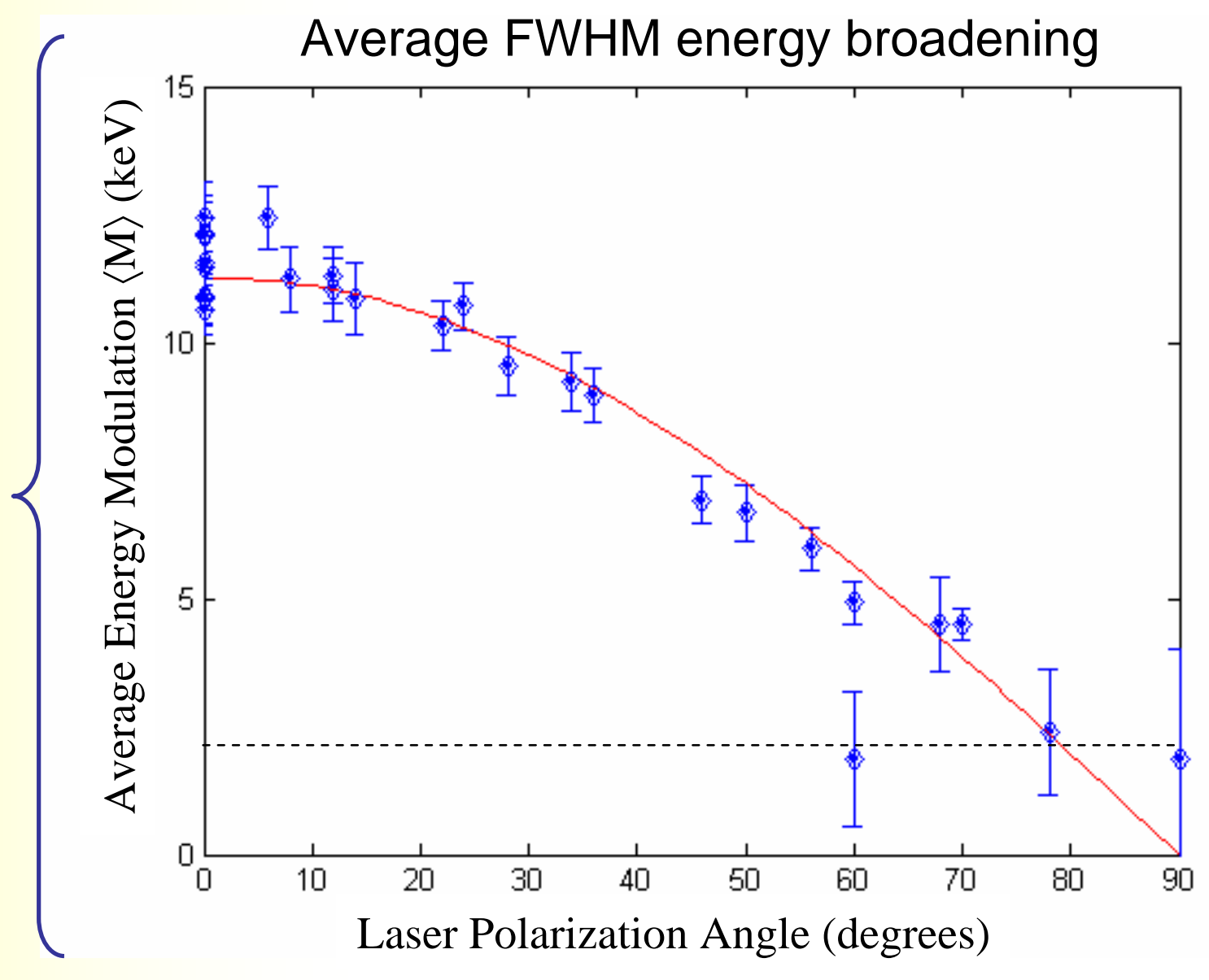




\section{Laser crossing angle dependence}

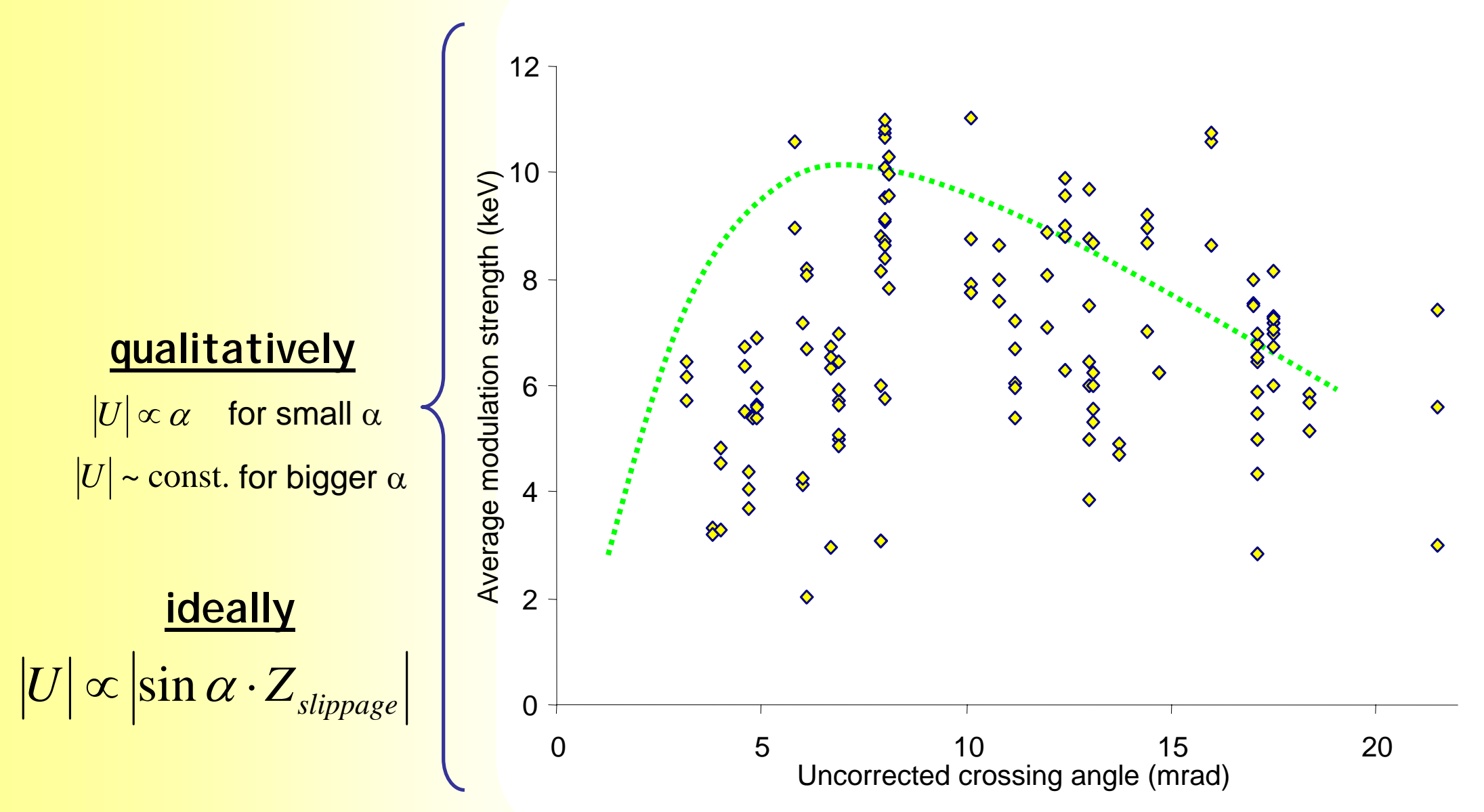

- me asure ment required realigning of laser and electron beam

- data set extended over several days

- electron beam conditions varied over this period of time 


\section{Did we do a modified plasma wakefield accelerator experiment?}

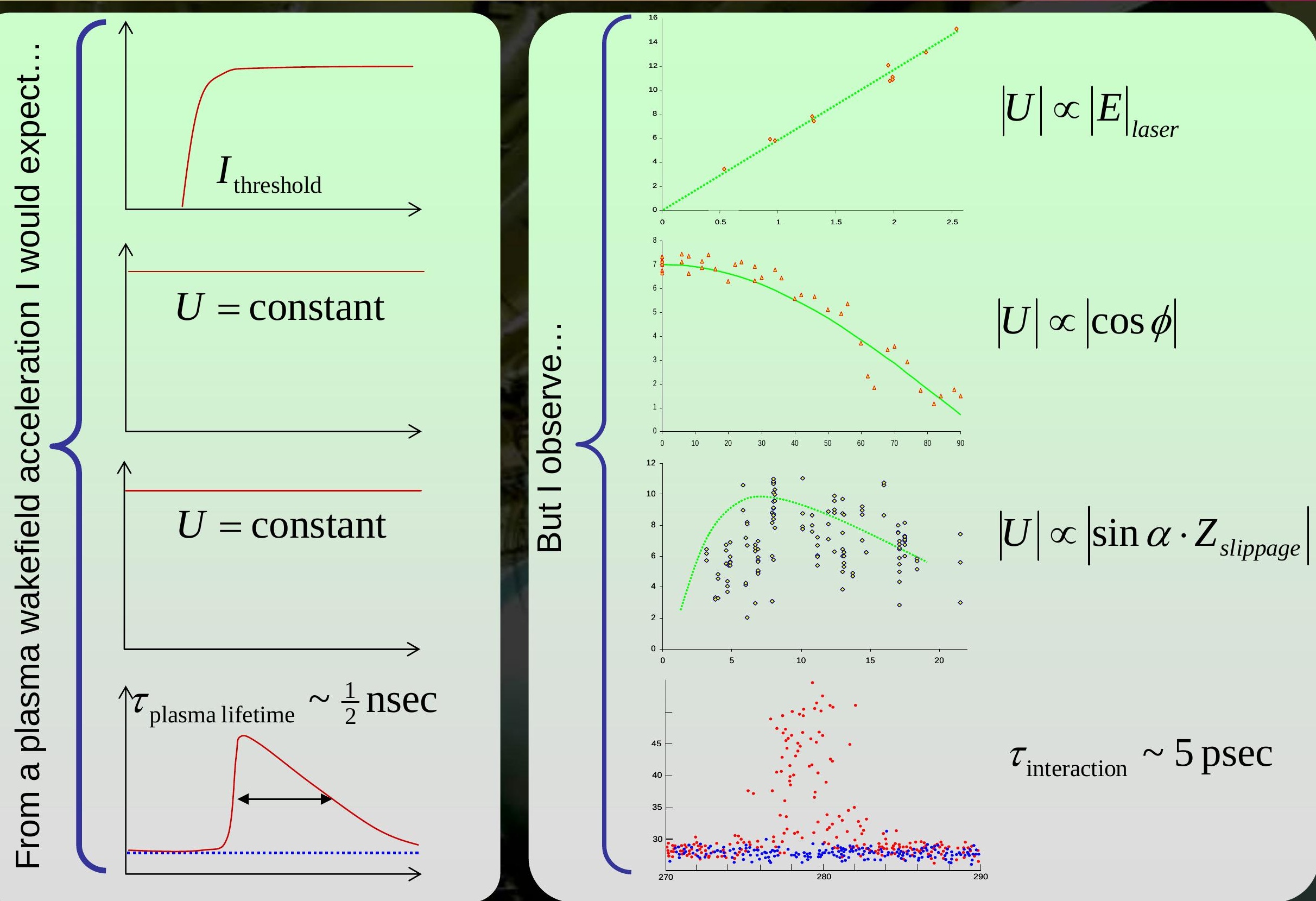




\section{Contents of the presentation}

\section{Overview}

- Motivation for laser-driven particle acceleration

- The physics concept

- Overview of the LEAP experiment

\section{The LEAP experiment}

- Important components of the LEAP experiment

- The simplified acceleration geometry

- The disposable boundary approach

- The insertion of an IFEL as a timing monitor

\section{Results}

- Confirmation of the Lawson-Woodward Theorem

- Proof of the linear dependence on the electric field

- Expected polarization dependence

- Expected slow dependence on laser crossing angle

4. Future experiments

- Test on different boundaries

- Measurements below the laser damage threshold

- E163 at SLAC

5. Summary 


\section{The E163 experiment}

Phase I: Single Cell Laser Acceleration

This is the continuation of the LEAP experiment

\section{objectives}

1. repetition of the tape boundary experiment verify

- laser power, polarization \&crossing angle

- boundary type

- reflected laser spot quality

2. switch to a dielectric single cell structure verify

- laser-electron interaction length

- laser crossing angle

- accelerator aperture size

•tolerances of the cell

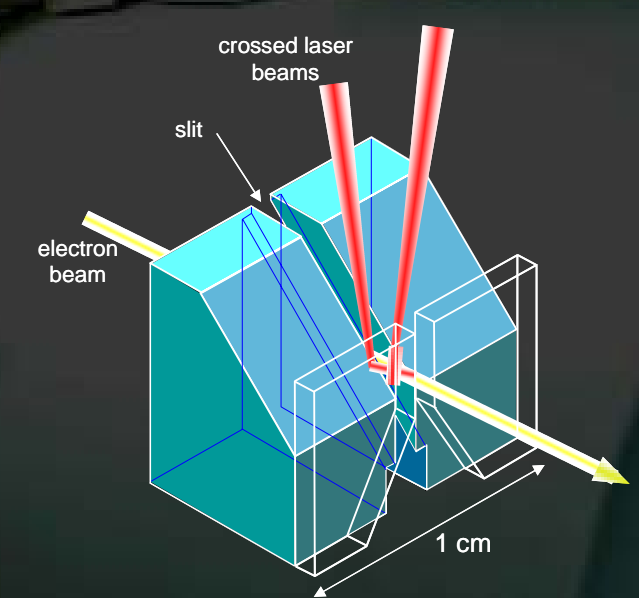


Phase II: Optical buncher-accelerator cellstaged experiments

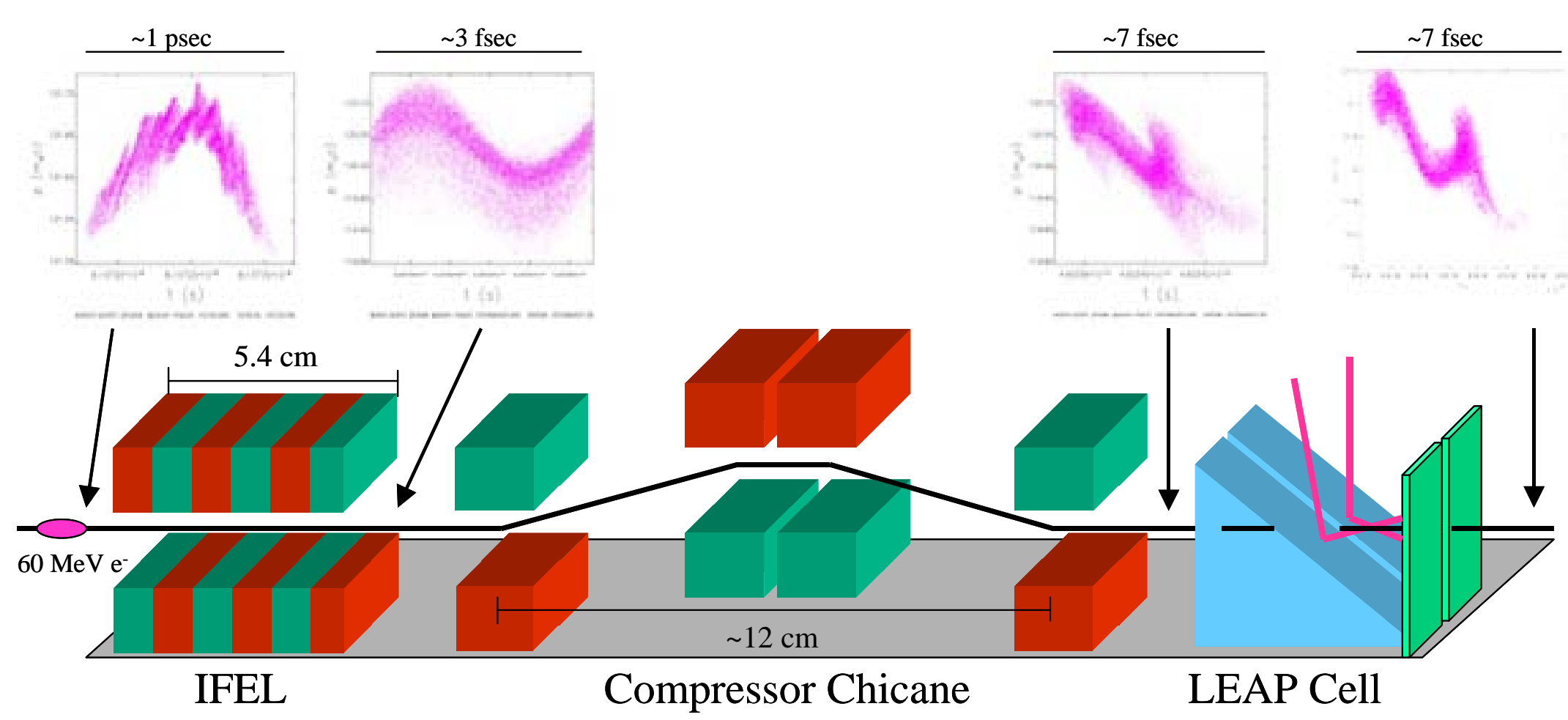

See publication by C.M. Sears et al, "High Harmonic IFEL at 800 nm“ (TPAE029) 


\section{The E163 experiment}

\section{Phase I I I: Guided-Mode Structures}

\section{(a)}

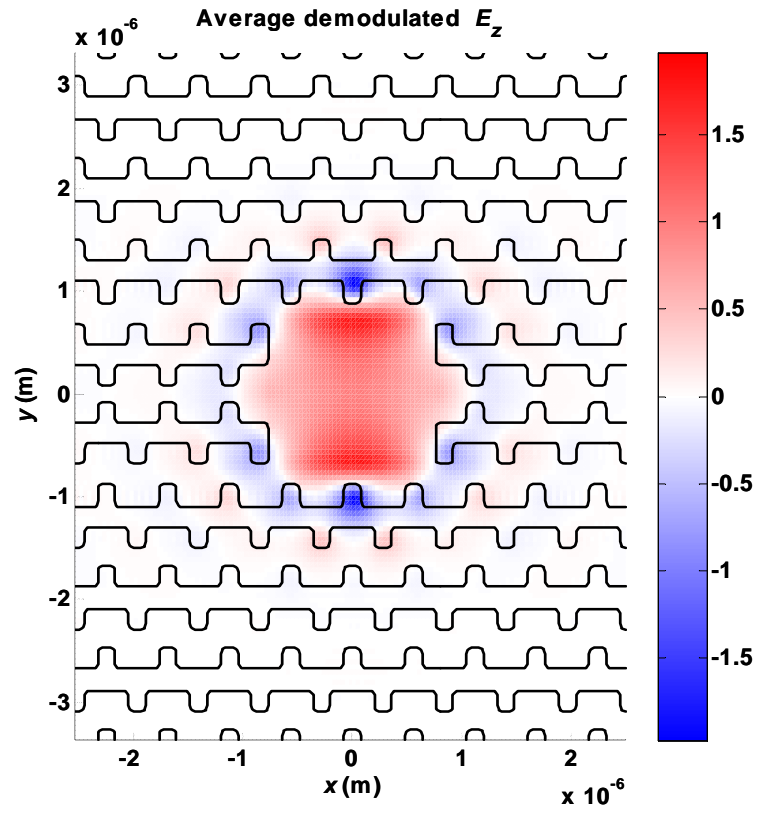

(b)

Defect radius $\mathrm{R}=0.52 \mathrm{a}$

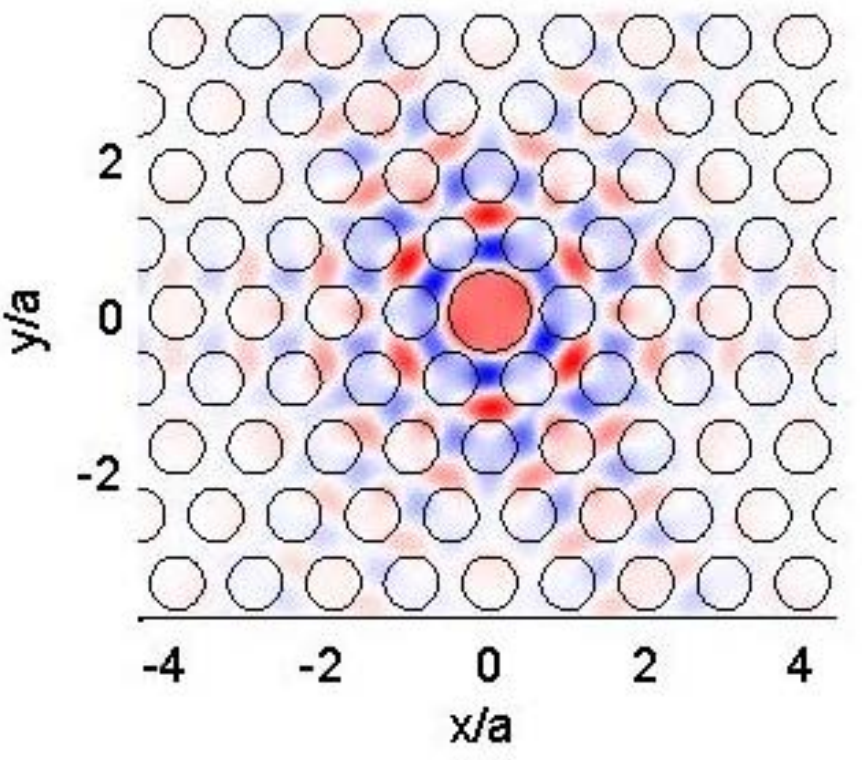

See publication by B. Cowan, "Photonic Crystal Laser-Driven Accelerator Structures" (TOPA010) 
- we have laser-accelerated electrons in vacuum

The laser wavelength was in the visible

This was an energy-modulation experiment We employed a disposable boundary

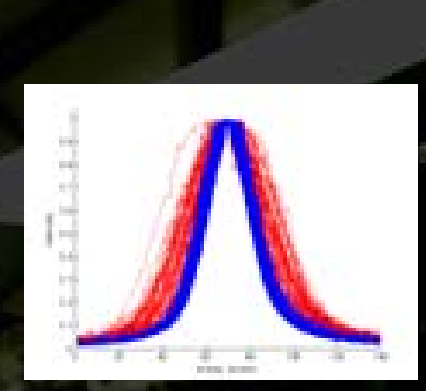

- the effect is consistent with laser driven particle acceleration in vacuum

It follows the Lawson-Woodward theorem

$$
\begin{aligned}
& U \leftrightarrow\left|E_{\text {laser }}\right| \\
& U \leftrightarrow|\cos \phi| \\
& U \leftrightarrow\left|\sin \alpha \cdot Z_{\text {slippage }}\right|
\end{aligned}
$$

- with the successful IFEL operation we have already started phase II of E-163 The results from the IFEL alone are new

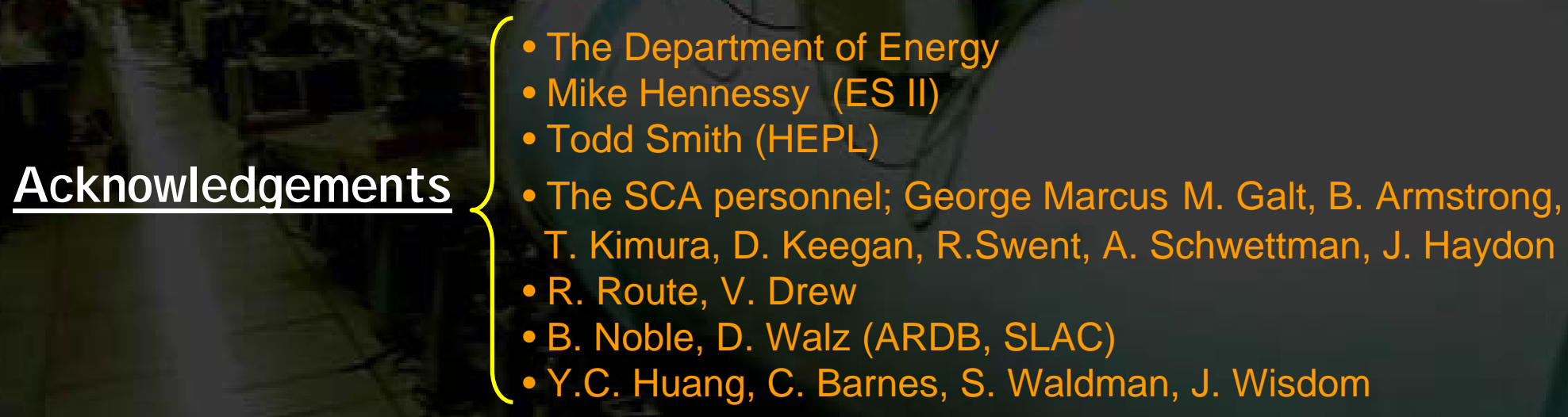




\section{Objective}

Develop materials and micro-machining technologies for integrated micro-elements for laser driven particle accelerators. Our focus is on magnetic and optical micro-elements.

$$
\text { Summary of Work }
$$

We have focused on micromachining techniques on silicon and have begun exploring ceramic materials as components for laser driven particle accelerator structures. In addition, we have carried out studies on radiation sensitivity of various candidate materials.

\section{Silic on micromachining}

\section{Advantages of $\mathrm{Si}$}

- advanced micromachining technology

- possibility for integrated electronic circuits

- optically transparent 1.5-10 $\mu \mathrm{m}$

- abundant and inexpensive

- high index of refraction

- good heat conductor

- radiation resistant

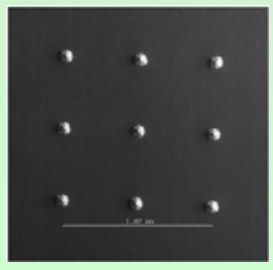

Sharp point-features

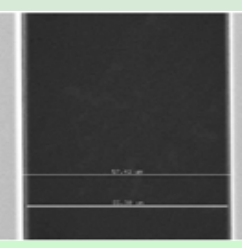

mm-deep trenches

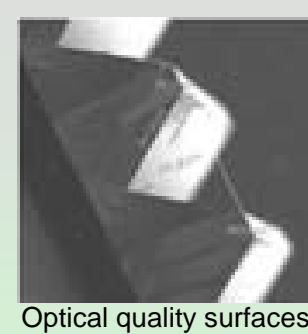

Problems

-limited geometry freedom with existing anisotropic etching techniques -semiconductor: $\rightarrow$ lower bandgap energy, easier multiphoton absorbtion and thus lower laser damage threshold
Desired Properties

of the materials

- high laser damage threshold

- radiation resistant

- high melting point

- good thermal conductivity

- optical transparency

- inexpensive, machineable

*E. Colby, G. Lum, T. Plettner, J. Spencer, "Gamma

Radiation Studies on Optical Materials", IEEE Trans. Nucl.

Sci. Vol. 49, No. 6, p. 2857-2867 (2002)

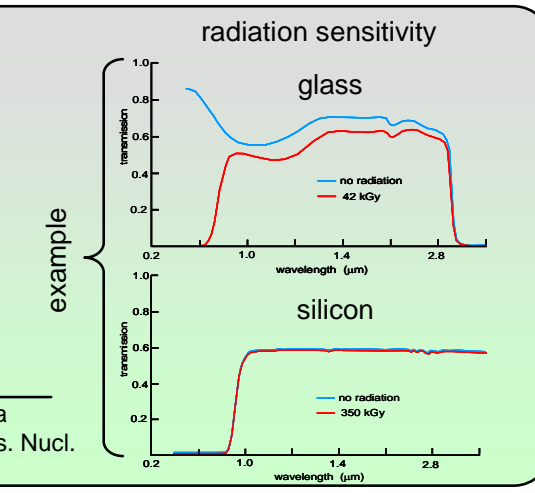

\section{Ceramics micromachining} Advantages of ceramics

- possibility for magnetic and optical materials from compatible substrates

- macroscopically amorphous: no restricted geometry limitations

- potentially higher degree of purity and less defect sites

- enhanced flexibility in mixing of different materials

- do not require expensive crystal growth step

- typical substrates like YAG: larger bad gap

$\rightarrow$ also transparent at visible wavelengths

$\rightarrow$ higher laser damage threshold

$\rightarrow$ higher melting points

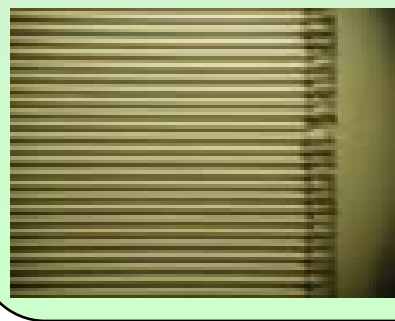

$50 \mu \mathrm{m}$ spacing pattern Imprinted with a Silicon preform

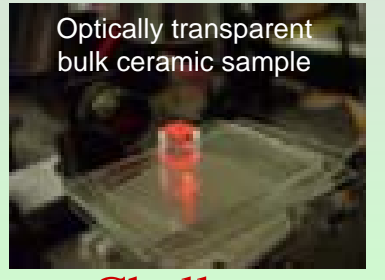

Challenges

- optical ceramics

fabrication technology

is a relatively new topic

- micromachining technology on ceramics 


\section{Optical Phase Locking of Modelocked Lasers for Particle Accelerators}

Present accomplisfiments -observed the comb offset from a commercial Ti:Sapphire modelocked laser

- isolated a comb offset error signal

-manipulated the comb offset with an electronic signal - constructed and tested a balanced cross-correlator -we are presently switching to a $1 \mathrm{~mm}$ Yb:glass fiber modelocked laser

Comb offset detection experiment

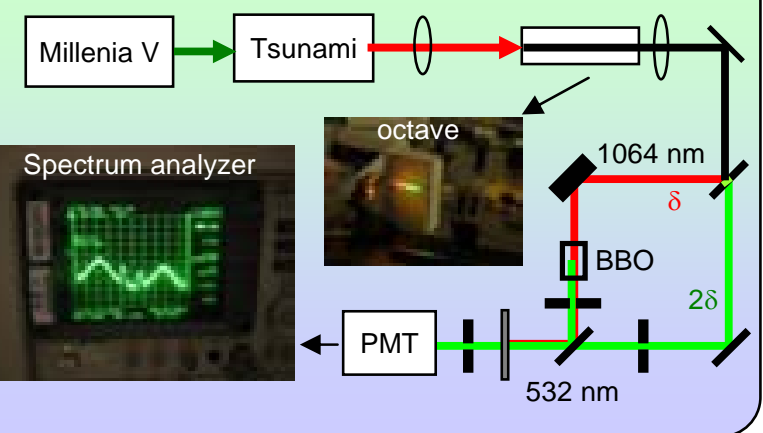

Beatnote detection circuitry PMT splitter bandpass
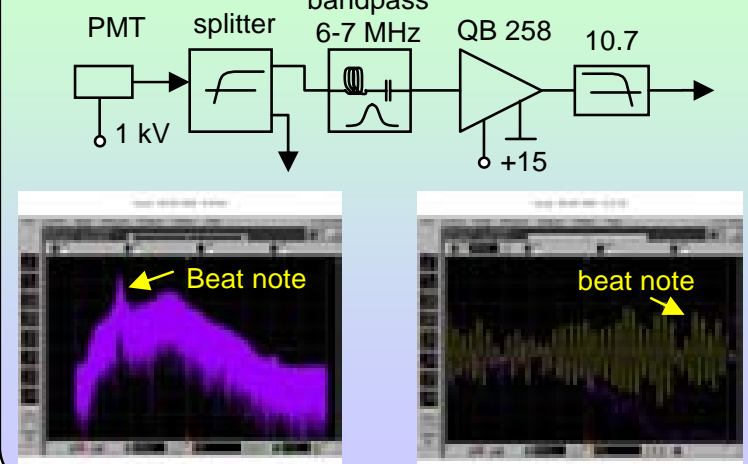

Two parameters for the control of the optical phase for modelocked lasers

Pulse envelope timing and carrier-to-envelope phase
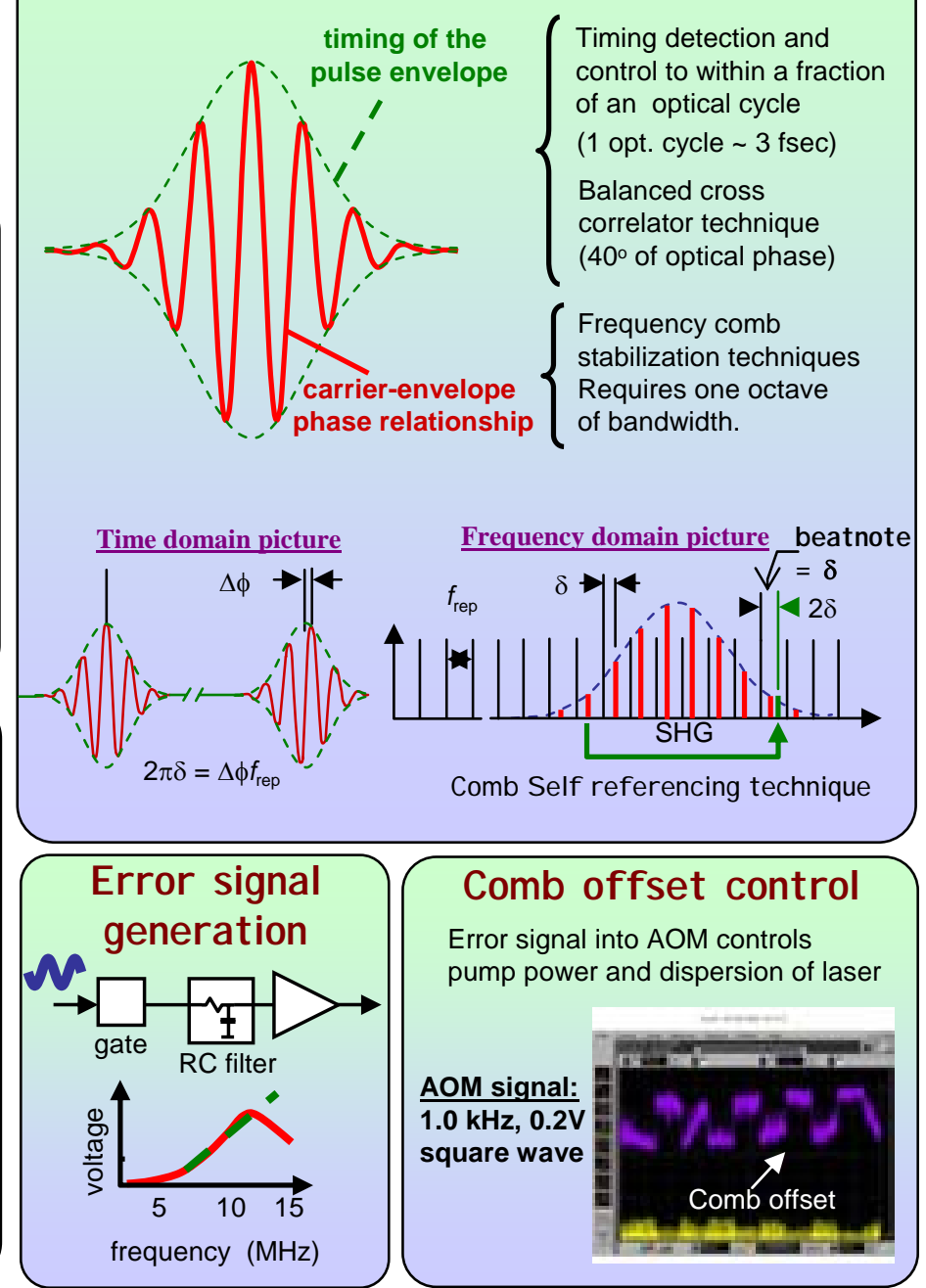

Comb offset control Error signal into AOM controls pump power and dispersion of laser

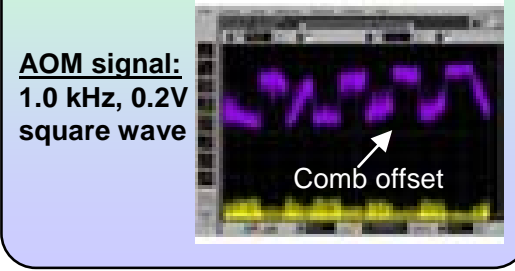

The Galanced cross.

correlator experiment
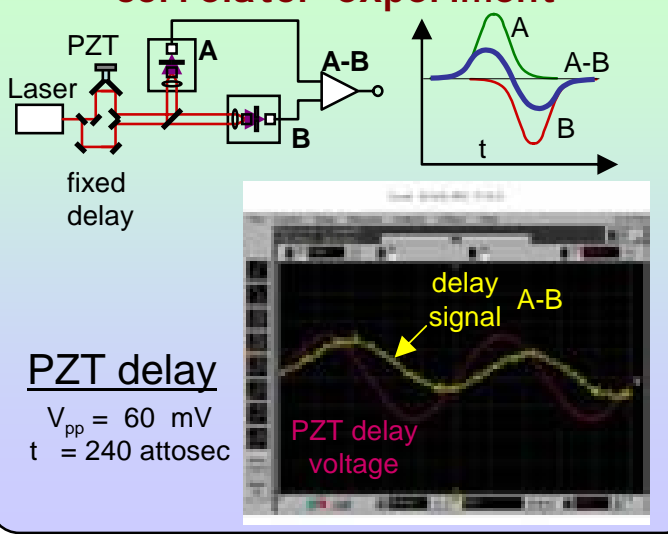

Future objectives

repeat these measurements with the fiber laser -feedback the comb error signal and stabilize the comb offset of a single laser

- construct a second identical fiber laser -synchronize the pulse envelopes of the lasers to sub-fsec with the balanced cross-corr. technique

- eliminate residual phase jitter with an external interferometer unit controlling a coptrabable puthedelay (e.g. a PZT) envelope to $\sim 40^{\circ}$ interferometer

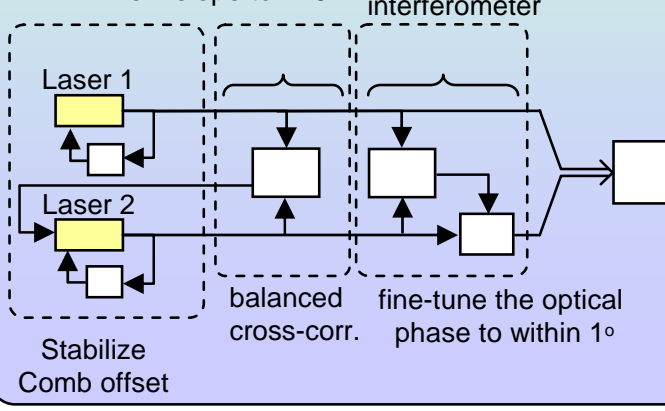

† Supported by DOEW grants DE-FG03-97ER41276 and DE-AC02-76SF00515. 


\section{Side Note on laser ablation dynamics}
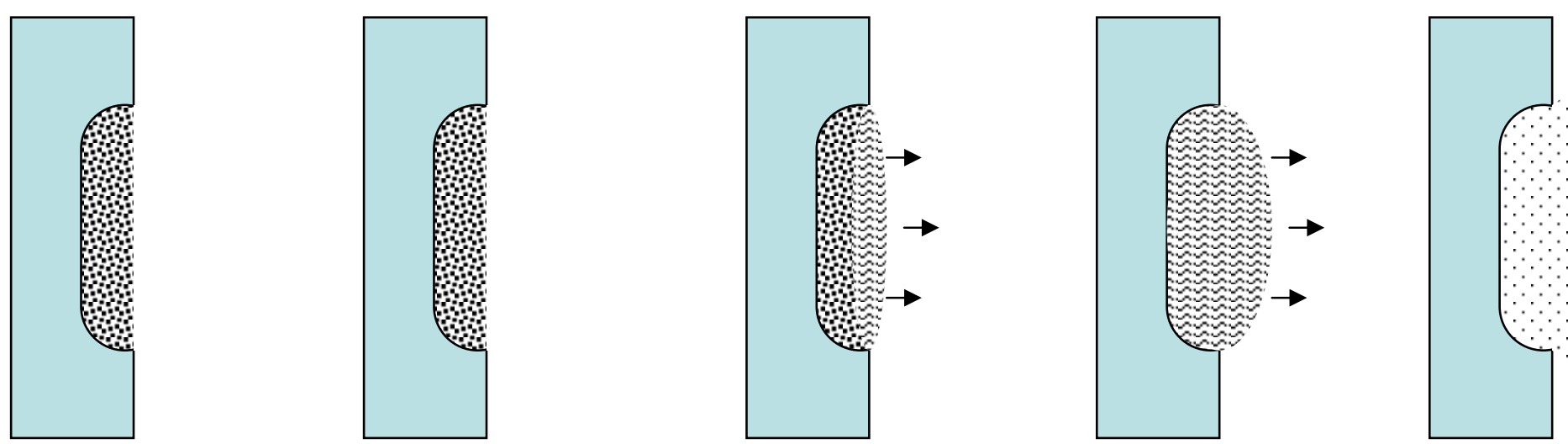

2 psec laser pulse
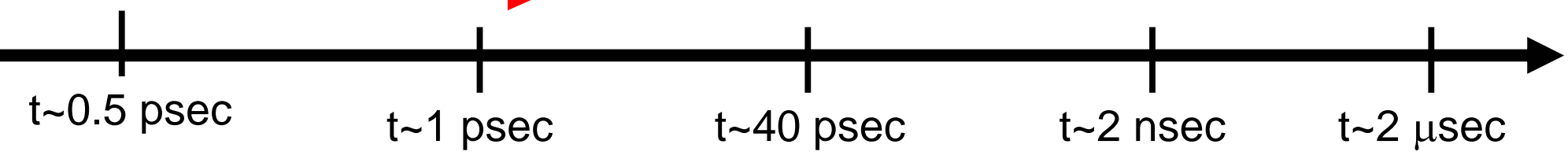

\section{excitation stage}

-excited solid

-formation of electron-

hole pairs

-Renormalization of

the band structure

-Change of the optical

properties

-Reduction of the

band gap

\section{transition stage \\ rarefaction \\ wave}

-Transformation into

a liquid sate

- 100 nm layer of

material with metallic

properties
-Low-density shock wave traveling at the speed of sound of the material

c $\sim 2500 \mathrm{~m} / \mathrm{sec}$

\section{expansion phase}

-Expansion of the ablation front

\section{ejection phase}

-Ejection of monomers and molecular clusters

During the passage of the laser and the electron beam the surface of the tape is a metallic high reflector

$$
N_{e} \sim 10^{22} / \mathrm{cc} .
$$




\section{Computation of the energy modulation strength}

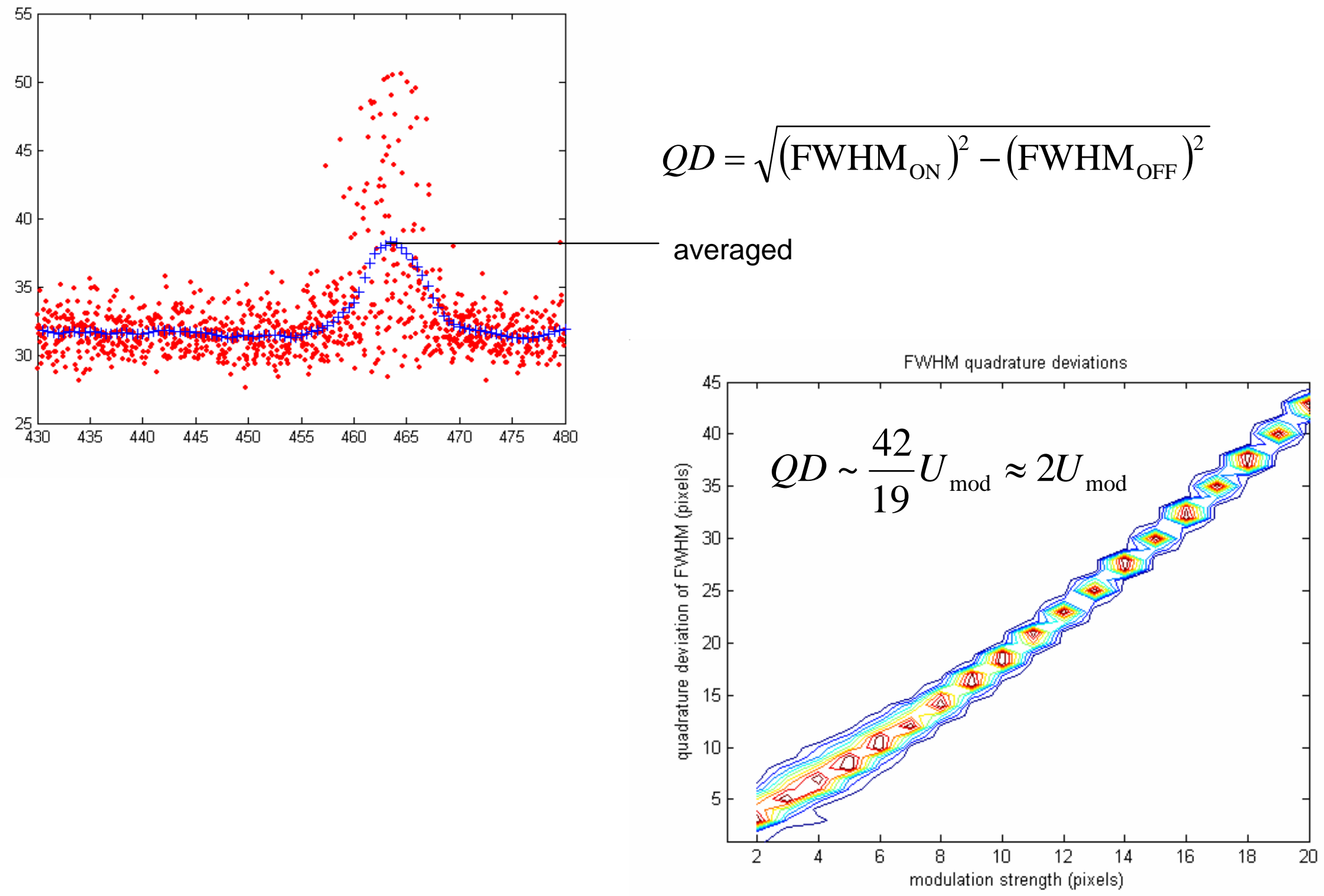

